\title{
TeVeS gets caught on caustics
}

\author{
Carlo R. Contaldi ${ }^{*}$ Toby Wiseman ${ }^{\dagger}$ and Benjamin Wither ${ }^{7}$ \\ Theoretical Physics, Blackett Laboratory, Imperial College, Prince Consort Road, London, SW7 2BZ, U.K.
}

(Dated: October 31, 2018)

\begin{abstract}
TeVeS uses a dynamical vector field with timelike unit norm constraint to specify a preferred local frame. When matter moves slowly in this frame - the so-called quasi-static regime - Modified Newtonian Dynamics (MoND) results. Theories with such vectors (such as Einstein-Aether) are prone to the vector dynamics forming singularities which render their classical evolution problematic. Here we analyse the dynamics of the vector in TeVeS in various situations. We begin by analytically showing that the vacuum solution of TeVeS forms caustic singularities under a large class of physically reasonably initial perturbations. This shows the classical evolution of TeVeS appears problematic in the absence of matter. We then consider matter by investigating black hole solutions. We find large classes of new black hole solutions with static geometries where the curves generated by the vector field are attracted to the black hole and may form caustics. We go on to consider the full dynamics with matter by numerically simulating, assuming spherical symmetry, the gravitational collapse of a scalar, and the evolution of an initially nearly static boson star. We find that in both cases our initial data evolves so that the vector field develops caustic singularities on a time scale of order the gravitational in-fall time. Having shown singularity formation is generic with or without matter, Bekenstein's original formulation of TeVeS appears dynamically problematic. We argue that by modifying the vector field kinetic terms to the more general form used by Einstein-Aether this problem may be avoided.
\end{abstract}

PACS numbers: 04.50.Kd,04.70.-s,04.25.D-,04.20.DW,04.70.Bw,97.60.Lf

\section{INTRODUCTION}

For many years the existence of dark matter has been postulated to reconcile a number of astrophysical and cosmological observations with our understanding of the laws of gravitation. Dark matter was originally introduced decades ago to explain the discrepancy between the rotation velocities of stars in the outskirts of galaxies and that predicted by the mass inferred from the amount of visible mass in the galaxy.

The success of the dark matter paradigm extends beyond galactic scales to cluster and indeed cosmological scales. Today we know that best fit models of structure formation apparently require a dark matter fraction much larger than the known baryon content of the universe to drive the growth of structure from kpc through to Gpc scales. The potential wells provided by a cold, dark matter (CDM) component also reconcile the amplitude of the acoustic peaks observed in the Cosmic Microwave Background (CMB) angular power spectrum [1, 2, 3, 4] with the known baryon content.

Dark matter also provides a simple explanation for the observed lensing of background galaxies by clusters along the line of sight. Recently the combination of optical, xray and lensing observations of the bullet cluster have yielded the most direct evidence to date in support of the picture where the gravitational mass of clusters is dominated by a dark matter component [5].

\footnotetext{
*Electronic address: c.contaldi@imperial.ac.uk

$\dagger$ Electronic address: t.wiseman@imperial.ac.uk

${ }_{\ddagger}$ Electronic address: benjamin.withers02@imperial.ac.uk
}

Taken as a whole the growing wealth of observations points clearly to a concordance $\Lambda \mathrm{CDM}$ cosmological model with a significant fraction of the critical energy density made up of CDM. The dark matter paradigm has stood the test of time remarkably well but significant questions remain. Many candidates for a dark matter particle exist ranging from massive neutrinos to more exotic weakly interacting extensions to the standard model. However dark matter has yet to be detected directly in the laboratory or indirectly possibly through the $\gamma$-ray signature of its decay in the centre of galaxies (this is required to avoid the concentration of dark matter observed in numerical simulations).

For these reasons an alternative approach to adding a dark matter component has been to consider whether the discrepancies between observations and general relativity in the low acceleration regime are an indication of the failure of the theory itself. This was the approach taken by Milgrom [6] who proposed a phenomenological modification to the acceleration equation which seems to fit well galactic rotation curves without the addition of any dark matter:

$$
\mu\left(|\mathbf{a}| / a_{0}\right) \mathbf{a}=-\nabla \Phi
$$

where $\Phi$ is the Newtonian potential, $\mu(x)$ is an arbitrary function with limits such that $\mu(x) \rightarrow 1$ in the strong acceleration regime $(x \gg 1)$. The constant $a_{0} \approx 10^{-10}$ $\mathrm{m} \mathrm{s}^{-2}$ determines the acceleration scale below which the Modified Newtonian Dynamics or MoND becomes relevant, and the above acceleration law receives non-linear corrections. While $\mu$ is potentially a free (monotonic) function, only the limits where its argument goes to zero or infinity affect the astrophysical phenomenology.

MoND has been successful in fitting the anomalous 
accelerations observed in galaxies and clusters (see e.g. [7] for a recent review). It also successfully predicts the Tully-Fisher relation correlating the luminosity of galaxies to the fourth power of the rotation velocity. However it remains a phenomenological modification of gravity with no underlying relativistic theory. In addition the simplest theory based on a modified action that reproduces Eq1depends explicitly on coordinates and breaks conservation laws. The fact that MoND has no underlying covariant theory has restricted its application for the purpose of comparison with other astrophysical and cosmological observations. For example, lensing predictions in both the strong and weak regimes cannot be formulated in MoND and this has left it unable to answer the criticism stemming from lensing mass reconstructions of galactic and cluster profiles which seem to suggest the existence of dark matter halos.

Recently however, Bekenstein [8], has put forward $\mathrm{TeVeS}$, a relativistic theory of gravity which reduces to MoND in the weak acceleration limit. In TeVeS the matter sector lives on a matter frame (MF) metric which maps 'disformally' to a second, Einstein or gravitational, frame (EF) metric via a dynamical scalar field $\phi$ and a dynamical vector field $A$. The addition of a scalar and vector degree of freedom are behind the name 'Tensor, Vector, and Scalar', or TeVeS theory. TeVeS builds on previous attempts to obtain a relativistic version of MoND which suffered from a number of inconsistencies involving the acausal propagation of physical degrees of freedom [9, 10]. TeVeS however was shown to be a fully causal theory for positive values of the additional scalar field.

The original motivation behind $\mathrm{TeVeS}$ was to build a theory with a fully consistent action which recovers the MoND behaviour in the weak acceleration limit. However, given it is a relativistic, metric theory of gravity and matter, it can do much more. In TeVeS it is possible to calculate geodesics in the presence of a matter sources which leads to lensing predictions [11]. It is also possible to show that it is compatible with the basic background cosmological observations such as age and distance measure observations [8]. The full framework of relativistic perturbation theory can be developed in TeVeS which makes comparison to the perturbed universe possible. Already the first calculations in this area have shown that the theory may be reconciled with CMB and Large Scale Structure (LSS) observations [12, 13, 14, 15], albeit with some fine tuning of the model ingredients. Attempts have also been made to explain the bullet cluster results within the TeVeS framework [16].

For TeVeS to be a successful theory it must also be shown to be consistent, and agree with observations, in the strong gravity regime. In exploring this end of the theory the potential is that it could be compared to astrophysical observations of compact objects such as neutron stars and black holes or at the solar system level with post Newtonian (ppn) corrections to planetary orbits $[8,17,18]$.
In order to have a modification of gravity dependent on acceleration, one must have a reference frame in which to measure that acceleration. The vector in TeVeS dynamically selects that reference frame, spontaneously breaking Lorentz invariance since it is constrained to have unit timelike norm. All types of matter see the same distorted metric so adding a preferred frame is not in conflict with weak equivalence principle tests. Only tests of gravitational dynamics can constrain the theory. MoND is recovered from TeVeS when matter moves nonrelativistically in the frame defined by the $\mathrm{TeVeS}$ vector, which has been termed the 'quasi-static' regime. The purpose of our work is to argue that this quasi-static regime will typically only exist for a short period of time, of order the gravitational in-fall time, after which the vector field develops a singularity and the theory cannot be classically evolved any further. Hence TeVeS even classically is dynamically sick in practice and recovery of MoND or even GR is impossible. Indeed here for simplicity we will focus on the large acceleration regime relevant on small scales (e.g. within the solar system) where the in-fall time scales are shortest.

This singular vector field behaviour is analogous to that in other modified gravity theories such as EinsteinÆther theory [19, 20] and Ghost Condensation 21, 22]. Einstein-Æther theory is much simpler than TeVeS, being simply Einstein gravity modified by adding a vector field, again with timelike unit norm constraint. The vector action is taken to be more general than that in TeVeS where it is simply that of a Maxwell field, but one may choose them to be the same. In this case (actually a theory written down earlier [23]), it is easy to show that the vector field generically develops singularities; classes of solutions exist where the integral curves of the vector are timelike geodesics moving in the spacetime geometry created by the matter. These geodesics fall into gravity potential wells and meet, and when they do so, the flow they define develops caustic singularities [19]. The vector field at these points becomes singular. It is for this reason that the Einstein-Æther literature focuses on other choices of the vector action than Maxwell type. Indeed while the ghost condensation theory has no vector, it is the integral curves of the gradient of the ghost scalar that form caustics. Since TeVeS is a considerably more complicated theory than Einstein-Æther, with complicated coupling of its vector and scalar to the matter, the vector behaviour and in particular whether it forms singularities could be very different. Our key result is that while in detail the dynamics of the vector is clearly different, it is still subject to the same singularity development as the Maxwell case of Einstein-Æther. However, we can play the same game as in Einstein-Æther theory, and by taking more general vector kinetic terms, we may avoid this behaviour, and as we show later, we still recover MoND for quasi-static systems.

The paper is organised as follows. In section $I \mathrm{~A}$ we review TeVeS theory and the field equations derived from the TeVeS action. We review the relation between TeVeS 
and the Einstein-Æther theory in section $\amalg \mathrm{B}$ where we introduce the problem of vector field caustic singularity formation. In section \we begin by showing analytically that in the absence of matter the TeVeS vacuum forms singularities under evolution of a class of initial perturbations. These are physically reasonable perturbations and we are able to precisely characterize the condition for the initial data developing to a singularity. We find that the condition is generic within the class of perturbations. Already this analysis indicates a fundamental problem with the TeVeS dynamics. However, phenomenologically it is the inclusion of matter in TeVeS that is of key interest, and hence we proceed to study whether such inclusion ameliorates or worsens the problem. We begin our study of matter in TeVeS in section III with an analytic treatment of black holes. Previous static black hole solutions of Giannios [17] and Sagi and Bekenstein [24] have a static vector field aligned with Killing time. However we find a large class of new solutions where the geometry is static but the vector field is in general dynamic. There exists a family of stationary solutions where the vector falls into the future horizon, but also dynamic solutions where caustics may form in the exterior of the black hole. The black hole provides a focus for the curves of the vector field and hence indicates that singularity formation is likely to be enhanced by compact matter sources. We then proceed in section [V with our study of matter by performing full numerical simulation of scalar collapse and the evolution of an initially quasi-static boson star. In both cases the evolution ends at a caustic, in the former outside of an apparent horizon, and in the latter near the surface of the star. Having given evidence that the dynamics of $\mathrm{TeVeS}$ is too pathological to provide a relativistic setting for the phenomenological theory of MoND, in section $\mathrm{V}$ we outline a modified TeVeS theory which may not suffer from caustic formation in the vector field. We explicitly show that MoND is again reproduced in the appropriate Newtonian limit. We argue that this modification is likely to affect many detailed phenomenological studies of TeVeS should therefore be included. We conclude with a summary and brief discussion of our main results in section VI

\section{A. TeVeS Action and Field Equations}

TeVeS is constructed using two metrics, the matter frame (MF) metric $\tilde{g}$, and the Einstein frame (EF) metric $g$. The two metrics are related through 'disformal relations' involving the extra scalar and vector fields $\phi$ and $A_{\mu}$

$$
\begin{aligned}
& \tilde{g}_{\alpha \beta}=e^{-2 \phi} g_{\alpha \beta}-2 A_{\alpha} A_{\beta} \sinh 2 \phi \\
& \tilde{g}^{\alpha \beta}=e^{2 \phi} g^{\alpha \beta}+2 A^{\alpha} A^{\beta} \sinh 2 \phi .
\end{aligned}
$$

The total action $S$ governing the dynamics in TeVeS can be split into separate components $S=S_{g+v}+S_{s}+S_{m}$, where

$$
\begin{aligned}
S_{g+v}= & \frac{1}{16 \pi G} \int\left[R-\frac{K}{2} F_{\mu \nu} F^{\mu \nu}+\right. \\
& \left.\left.\lambda\left(A^{2}+1\right)\right]\right)(-g)^{1 / 2} d^{4} x,
\end{aligned}
$$

where $g$ is the determinant of the EF metric, $R$ is the scalar curvature, $G$ is the gravitational constant and $F_{\mu \nu}=A_{[\mu, \nu]}=A_{\mu, \nu}-A_{\nu, \mu}$. The Lagrange multiplier $\lambda$ enforces the timelike, unit norm constraint on the vector field

$$
g^{\mu \nu} A_{\mu} A_{\nu}=-1
$$

The scalar field action is given by

$$
\begin{aligned}
S_{s}= & -\frac{1}{2} \int\left[\sigma^{2}\left(g^{\alpha \beta}-A^{\alpha} A^{\beta}\right) \phi_{, \alpha} \phi_{, \beta}+\right. \\
& \left.\frac{1}{2} G \ell^{-2} \sigma^{4} \mathcal{F}\left(\kappa G \sigma^{2}\right)\right](-g)^{1 / 2} d^{4} x,
\end{aligned}
$$

where $\sigma$ is a non-dynamical scalar field and $\mathcal{F}\left(\kappa G \sigma^{2}\right)$ is a dimensionless function whose behaviour is determined by requiring GR and MoND to be recovered in the appropriate dynamical limits. TeVeS introduces three new parameters; the two dimensionless constants $\kappa$ and $K$ and a third parameter $l$ with units of length.

Finally the matter action $S_{m}$ is built using the MF metric as

$$
S_{m}=\int L\left[\tilde{g}, \chi^{A}, \partial \chi^{A}\right](-\tilde{g})^{1 / 2} d^{4} x
$$

for a collection of matter fields $\chi^{A}$. Thus all matter fields are coupled to the same MF metric and test particles follow the same geodesics. This ensures the weak equivalence principle is satisfied and the theory is not in conflict with fifth force measurements.

Varying with respect to $g$, and recalling $\tilde{g}=\tilde{g}(g, A, \phi)$ gives the Einstein equations

$$
G_{\alpha \beta}=8 \pi G\left[\tilde{T}_{\alpha \beta}+\left(1-e^{-4 \phi}\right) A^{\mu} \tilde{T}_{\mu(\alpha} A_{\beta)}+\tau_{\alpha \beta}\right]+\Theta_{\alpha \beta},
$$

where $\tilde{T}_{\mu(\alpha} A_{, \beta)}=\tilde{T}_{\mu \alpha} A_{\beta}+\tilde{T}_{\mu \beta} A_{\alpha}, G_{\alpha \beta}$ is the Einstein tensor, $\tilde{T}_{\alpha \beta}$ is the energy momentum tensor of the matter components defined in terms of the MF metric $\tilde{g}_{\alpha \beta}$ and 


$$
\tau_{\alpha \beta} \equiv \sigma^{2}\left[\phi_{, \alpha} \phi_{, \beta}-\frac{1}{2} g^{\mu \nu} \phi_{, \mu} \phi_{, \nu} g_{\alpha \beta}-A^{\mu} \phi_{, \mu}\left(A_{(\alpha} \phi_{, \beta}-\frac{1}{2} A^{\nu} \phi_{, \nu} g_{\alpha \beta}\right)\right]-\frac{1}{4} G \ell^{-2} \sigma^{4} \mathcal{F}\left(\kappa G \sigma^{2}\right) g_{\alpha \beta}
$$

$$
\Theta_{\alpha \beta} \equiv K\left(F_{\alpha}{ }^{\mu} F_{\beta \mu}-\frac{1}{4} g_{\alpha \beta} F^{2}\right)-\lambda A_{\alpha} A_{\beta} .
$$

Variation with respect to the scalar field $\sigma$ yields a relation between $\sigma$ and $\phi_{, \alpha}$ involving $\mathcal{F}$. The specific choice of $\mathcal{F}$ determines the exact behaviour of the theory in the weak acceleration regime and is relevant for the MoND and cosmological behaviour of TeVeS. The regime of interest for this work is one where the acceleration is much stronger than the MoND acceleration scale $a_{0}$. In this case the MoND function $\mu\left(|a| / a_{0}\right) \rightarrow 1$, which is equivalent to a limit on the argument of the free function $\mathcal{F}$,

$$
\sigma^{2} \rightarrow \frac{1}{\kappa G}
$$

For any suitable function choice, $\mathcal{F}$ diverges logarithmically in $(\mu-1)$ in this limit. The contribution of $\mathcal{F}$ to the field equations (8) however is suppressed by a factor $(\mu-1)$ relative to other terms, and so when $\mu \sim 1$ it may be neglected [8, 17, 24]. Thus our results will be insensitive to any particular choice of $\mathcal{F}$ and we drop the term in the following. Finally variation with respect to the scalar gives

$$
\begin{array}{r}
{\left[\left(g^{\alpha \beta}-A^{\alpha} A^{\beta}\right) \phi, \alpha\right]_{; \beta}=} \\
\kappa G\left[g^{\alpha \beta}+\left(1+e^{-4 \phi}\right) A^{\alpha} A^{\beta}\right] \tilde{T}_{\alpha \beta},
\end{array}
$$

and for the vector we have

$$
\begin{array}{r}
K \nabla_{\beta} F^{\beta \alpha}+\lambda A^{\alpha}+\frac{8 \pi}{\kappa} A^{\beta} \phi_{, \beta} g^{\alpha \gamma} \phi_{, \gamma}= \\
8 \pi G\left(1-e^{-4 \phi}\right) g^{\alpha \mu} A^{\beta} \tilde{T}_{\mu \beta} .
\end{array}
$$

As stated here TeVeS is a classical phenomenological theory. The somewhat Baroque form for the Lagrangian leads to the obvious concern that the theory is not stable to quantum corrections. Attempts have been made to study a UV origin from String theory [25, 26], and there are certainly many interesting questions in these directions which we do not consider here.

It is also worth mentioning that the TeVeS theory itself been generalized by various authors [15, 27, 28, 29, 30] and it would be interesting to consider the formation of caustics which we study here in these modified versions of the theory.

\section{B. Ether theory, its relation to TeVeS, and problems with its vector field dynamics}

Another theory of aether field dynamics is EinsteinÆther theory [19] - an effective field theory designed to investigate the effects of Lorentz violation in a fully covariant setting. It has the action,

$$
\begin{aligned}
& \frac{1}{16 \pi G} \int\left[R+K_{\mu \nu}^{\alpha \beta} \nabla_{\alpha} A^{\mu} \nabla_{\beta} A^{\nu}+\right. \\
& \left.\lambda\left(A^{2}+1\right)\right](-g)^{1 / 2}+\int \mathcal{L}_{\text {matter }}[g],
\end{aligned}
$$

where $K^{\alpha \beta}$ provides the most general kinetic term for $A$ which is diffeomorphism invariant, quadratic in derivatives and (preemptively) consistent with the $A^{2}=-1$ constraint. Specifically,

$$
K_{\mu \nu}^{\alpha \beta}=c_{1} g^{\alpha \beta} g_{\mu \nu}+c_{2} \delta_{\mu}^{\alpha} \delta_{\nu}^{\beta}+c_{3} \delta_{\nu}^{\alpha} \delta_{\mu}^{\beta}+c_{4} A^{\alpha} A^{\beta} g_{\mu \nu}
$$

This kinetic term is the usual Maxwell case when $c_{+} \equiv$ $c_{1}+c_{3}=0, c 4=c 2=0, c_{-} \equiv c_{1}-c_{3}<0$. EinsteinEther theory is actually a truncation of TeVeS in the absence of matter, where we may consistently set the scalar to zero and then $c_{-}=-2 K$ - however obviously phenomenologically this is not the regime of interest for TeVeS where the coupling to matter and the non-zero scalar are crucial.

Following Jacobson \& Mattingly [19] it is easy to see that the Maxwell case of Einstein-Æther is pathological. To any solution of Einstein gravity coupled to matter, we may simply add a vector field obeying the equations,

$$
F_{\mu \nu}=0, \quad A^{2}=-1
$$

and this will then solve the full Einstein-Æther equations for that matter since the vector and constraint contribute nothing to the stress energy. Note that the vacuum, the Minkowski geometry and $A^{\mu}=\left(\partial_{t}\right)^{\mu}$, is in this class of solutions. Generally the solution is given by,

$$
A_{\mu}=\partial_{\mu} \chi, \quad(\partial \chi)^{2}=-1
$$

where the latter equation is a p.d.e., first order in time,

$\partial_{t} \chi=\frac{1}{\left(-g^{t t}\right)}\left(g^{t i} \partial_{i} \chi-\sqrt{\left(-g^{t t}\right)\left(1+\partial^{i} \chi \partial_{i} \chi\right)+\left(g^{t i} \partial_{i} \chi\right)^{2}}\right)$

with $i=1, \ldots, 3$, which can evolve $\chi$ in time from an initial Cauchy surface. We have taken the choice of root above since we wish $A^{\mu}$ to be a future directed vector field. Hence the data for the solutions can be characterized by the function $\chi(t=0, x)$. Now, $A^{\nu} \nabla_{\nu} A_{\mu}=$ $A^{\nu} \nabla_{\mu} A_{\nu}=0$ using both relations in (16). Hence integral curves of the vector field $A$ are simply timelike geodesics.

Suppose we consider a static star as a matter source. Then the solution above will have families of vector fields 
with different initial directions, but all will have integral curves that fall in towards the gravitational potential well and will meet each other in a timescale of order the gravitational in-fall time. Since these are integral curves of the vector field, when they meet they result in a caustic singularity where the value of the vector is ill-defined. Indeed, even in the absence of matter and with the Minkowski spacetime geometry it is possible to have singular behaviour. Such a solution is illustrated in figure 1 .

This simple argument shows for solutions with $F_{\mu \nu}=0$ that caustic singularities generically occur in the presence of gravitational potential wells. It seems reasonable that singularities will also occur in solutions where $F_{\mu \nu} \neq 0$. Whilst there are no general arguments, in specific cases with $F_{\mu \nu} \neq 0$ singularity formation has been shown by Clayton [20].

It is for these reasons that the Einstein-Æther literature does not consider the Maxwell vector kinetic term [31]. Interestingly there is little rigorous understanding for what choices of parameters $c_{i}$ do give well behaved vector dynamics. Clearly a caustic singularity is signalled by the divergence of the vector field becoming infinite. Hence it is expected that by adding the term $c_{2}$ appropriately, which directly energetically weights this divergence, one can dynamically suppress singularities. For example, taking the Maxwell case together with the additional term $c_{2}$, we obtain a vector equation of motion,

$$
\left(\delta_{\mu}^{\alpha}+A^{\alpha} A_{\mu}\right)\left[\partial_{\nu} F^{\nu \mu}+c_{2} \partial^{\mu}(\partial \cdot A)\right]=0 .
$$

We note that when $c_{2}=1$ the equation of motion is essentially the wave equation, and hence in a regular geometry we would certainly not expect singular behaviour. How large $c_{2}$ should be to avoid singularities is an interesting open problem.

Ignoring gravity and matter, we simply plot a vector solution to the above equation in $1+1$ flat space in figures 11 and 2. The left frame is for $c_{2}=0$, the right for $c_{2}=0.2$, and both have the same initial data which satisfies $F_{\mu \nu}=0$. Note that the right frame cannot be lifted simply to a solution of Einstein-Æther since for $c_{2}=0.2, F_{\mu \nu}$ will not remain zero, and the vector necessarily contributes to the gravitational stress tensor. However, clearly by eye we see a change in behaviour, where the small amount of positive $c_{2}$ avoids the caustic, leading to an asymptotic vector solution which is aligned with time.

\section{VECTOR FIELD DYNAMICS IN THE ABSENCE OF MATTER}

Whilst in Einstein-Æther we can exhibit the large class of solutions of the vector field in (17) which lead to caustic singularities, it is far from obvious that the same occurs in TeVeS. For these solutions, $F_{\mu \nu}=0$ and this leads geometrically to the integral curves of the vector simply

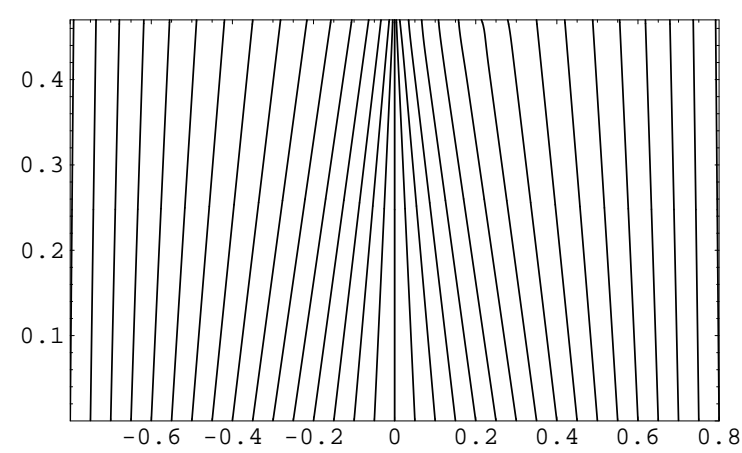

FIG. 1: Flat Space evolution of a Gaussian perturbation to the radial component of the vector field for the Einstein-Maxwell case $\left(c_{2}=0\right.$, no divergence term included). The solution displays caustic instabilities.

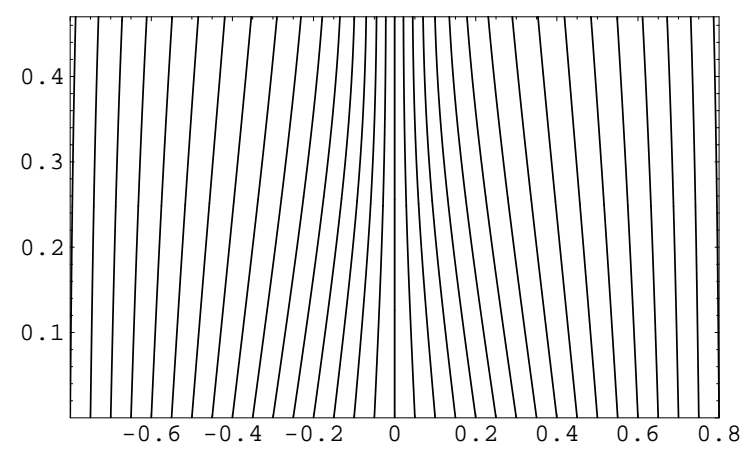

FIG. 2: Flat Space Same as figure 1 but for $c_{2}=0.2$ i.e. a divergence term included in the kinetic part of the EinsteinÆther vector action. The addition of a divergence term suppresses the formation of caustics.

being geodesics that fall into the gravitational wells created by the matter. However in $\mathrm{TeVeS}$ there is direct coupling between the vector and matter, and hence in the presence of matter one can not have $F_{\mu \nu}=0$. If $F_{\mu \nu} \neq 0$, then the curves of the vector do not follow geodesics, and we cannot argue that they must cross forming caustic singularities, even if we suspect they might.

However we can make some analytic progress in the absence of matter so $\tilde{T}_{\mu \nu}=0$. Then we may consistently truncate to solutions with constant scalar field. Initial data with constant scalar and vanishing scalar time derivative, i.e. $\partial_{\mu} \phi=0$ on an initial Cauchy surface, evolves to have constant scalar.

We stated earlier that we are interested in the strong acceleration regime, for example we look at the dynamics on solar system scales or smaller. We denote this scale of interest by $L$. Since the acceleration regime is actually determined by the scalar gradient, a precisely constant scalar corresponds to exactly the opposite, the low acceleration MOND regime, even if all other dynamical fields have characteristic scales given by $L$. However, we are 
envisaging a physical situation in which the TeVeS scalar has long wavelength fluctuations set by surrounding matter, for example set by the galaxy in which the region of interest is embedded. These fluctuations will be taken to have gradient large enough to place our region of interest into the Newtonian regime, which translates to the condition that the scalar should vary on lengths set by the TeVeS scale $\ell$. In Appendix A, we show that given this vast separation of scales, $L \ll \ell$, a solution to the TeVeS equations where we instead take exactly constant scalar, ignore $\mathcal{F}$ and set $\mu=1$, is a good approximation to the full TeVeS equations, within the scale of interest $L$. 62]

For a constant scalar the vector equation reduces to

$$
K \nabla_{\beta} F^{\beta \alpha}+\lambda A^{\alpha}=0 .
$$

Consider starting with initial data on a Cauchy surface $\Sigma$ at $t=0$ where $F_{\mu \nu}=0$. Since $A^{\mu}$ is timelike, the $t$ component $A^{t}$ cannot vanish, and hence the $t$ component of the above vector equation sets $\lambda=0$ on this initial data surface. However, the remaining components $i=$ $1 \ldots 3$ then determine $\partial_{t} F^{t i}=0$ at $t=0$. Furthermore the Bianchi identity for $F_{\mu \nu}, \nabla_{[\mu} F_{\nu \alpha]}=0$ implies that $\partial_{t} F_{i j}=0$ at $t=0$. Together these imply that $\partial_{t} F_{\mu \nu}=0$ and so $F_{\mu \nu}$ remains zero when evolved off the surface $\Sigma$. Hence we see that starting with initial data $\partial_{\mu} \phi=F_{\mu \nu}=$ 0 on $\Sigma$ implies (in the absence of matter) that the scalar is constant, $F_{\mu \nu}=0$ and $\lambda=0$ for all $t$. As discussed above, for $F_{\mu \nu}=0$ the vector can then be written as $A_{\mu}=\partial_{\mu} \chi$ with the timelike constraint $A^{\mu} A_{\mu}=-1$ giving the p.d.e. in equation (18) which can be used to evolve $\chi$. Hence the initial data for the vector can be parametrized by the function $\chi$ on $\Sigma$ which determines the direction of the vector on $\Sigma$.

The dynamics of $\mathrm{TeVeS}$ in this truncation are the same as those of Einstein-Æther with Maxwell kinetic term and no matter. Hence as we claimed above, for $F_{\mu \nu}=0$ where vector integral curves are timelike geodesics, we should expect to be able to form caustics. While this is true, and indeed figure 1 gives an example in $1+1$ for the Minkowski geometry, there is no matter to focus the geodesics and hence it isn't obvious how generic caustic formation would be. If we start with initial data in this class of solution - i.e. suitable data for the metric, together with the function $\chi$ which specifies initial data for the vector - are the initial data that develop to a singularity generic, or a special case? We now address this by precisely characterizing when initial data will form a caustic. We note that while our analysis is given in the context of TeVeS, precisely the same argument can be made in the context of Einstein-Æther theory, although we know of no previous literature doing so.

Hence we consider $\mathrm{TeVeS}$ in the absence of matter, with constant scalar, and with $F_{\mu \nu}=0$. We note that the TeVeS vacuum, with Minkowski geometry and $A^{\mu}=\left(\partial_{t}\right)^{\mu}$ is in this class, and hence we may regard the class as a restricted (although not necessarily small) deformation of the TeVeS vacuum. The equation for the metric immediately gives $R_{\mu \nu}=0$. Thus the class covers gravity wave spacetimes and black hole exteriors (with constant scalar).

We now briefly review some basic facts in GR. For a congruence of timelike geodesics, parametrized by proper time $\tau$ with tangent vector field $\xi^{\mu}$, with $\xi^{\mu} \xi_{\mu}=-1$, we may define a tensor field

$$
B_{\mu \nu}=\nabla_{\nu} \xi_{\mu},
$$

which then satisfies $B_{\mu \nu} \xi^{\mu}=B_{\nu \mu} \xi^{\mu}=0$. We define the expansion $\theta$, shear $\sigma_{\mu \nu}$, and twist $\omega_{\mu \nu}$ as,

$$
\begin{aligned}
\theta & =B^{\mu \nu} h_{\mu \nu}, \\
\sigma_{\mu \nu} & =\frac{1}{2} B_{(\mu \nu)}-\frac{1}{3} \theta h_{\mu \nu}, \\
\omega_{\mu \nu} & =\frac{1}{2} B_{[\mu \nu]},
\end{aligned}
$$

where $h_{\mu \nu}=g_{\mu \nu}+\xi_{\mu} \xi_{\nu}$ is the projector onto the tangent space orthogonal to the timelike geodesics. Then Raychaudhuri's equation is,

$$
\frac{d \theta}{d \tau}=-\frac{1}{3} \theta^{2}-\sigma_{\mu \nu} \sigma^{\mu \nu}-\omega_{\mu \nu} \omega^{\mu \nu}-R_{\mu \nu} \xi^{\mu} \xi^{\nu} .
$$

Now we consider applying this result to our situation. Recall that since our solutions have $F_{\mu \nu}=0$, then the integral curves of $A^{\mu}$ are timelike geodesics, and moreover the tangent vector $A^{\mu}$ has unit norm. Hence we may take the $\xi^{\mu}$ above to be $A^{\mu}$. Then since $F_{\mu \nu}=0$ the twist $\omega_{\mu \nu}$ vanishes, and hence the congruence is hypersurface orthogonal. Furthermore we have $R_{\mu \nu}=0$, and using the fact that $\sigma_{\mu \nu} \sigma^{\mu \nu} \geq 0$ therefore arrive at the expression,

$$
\frac{d \theta}{d \tau} \leq-\frac{1}{3} \theta^{2}
$$

where $\theta=\left(\nabla_{\mu} A_{\nu}\right)\left(g^{\mu \nu}+A^{\mu} A^{\nu}\right)=\nabla \cdot A$ since $A^{\mu} A_{\mu}=$ -1 . Thus we have the result,

$$
(\nabla \cdot A)^{-1}(\tau) \geq(\nabla \cdot A)_{0}^{-1}+\frac{1}{3} \tau,
$$

along a geodesic with $\nabla \cdot A=(\nabla \cdot A)_{0}$ at the point where the geodesic intersects the initial Cauchy surface $\Sigma$. So we conclude that if $\nabla \cdot A<0$ anywhere on the initial hypersurface $\sigma$, within a proper time $-3(\nabla \cdot A)_{0}^{-1}, \nabla \cdot A$ diverges, signaling that the geodesic congruence ends at a caustic singularity.

In summary, we have obtained the following result: In the absence of matter smooth initial data with $\partial_{\mu} \phi=$ $F_{\mu \nu}=0$ on a spacelike hypersurface $\Sigma$ will evolve to form a caustic singularity if $\nabla \cdot A<0$ anywhere on $\Sigma$. Note that while these are solutions with exactly constant scalar, the timescale of caustic formation is set by $L$ and so they will still be good approximations to the TeVeS equations in the strong acceleration regime, as discussed in Appendix A

Whilst this class of solutions is clearly restricted it is still physically reasonable and in particular includes initial data close to the TeVeS vacuum. The initial data 
includes the initial data for the metric and for the vector, the function $\chi$ on $\Sigma$. The condition that $\nabla \cdot A<0$ at any point on the initial data surface is very weak, and certainly generic within our restricted class. For example, consider the small perturbation from the TeVeS vacuum where the metric is taken to be Minkowski and the vector near the initial surface $\Sigma$ at $t=0$ is given by $\chi=-t+\delta \chi$, for small $\delta \chi$. Then the singularity condition on $\Sigma,(\nabla \cdot A) \simeq \nabla_{i}^{2} \delta \chi<0$ will be generically satisfied in the region surrounding a maximum in $\chi$ on $\Sigma$.

Hence our result very clearly highlights the fact that caustic singularities do indeed occur in TeVeS. Note that we have bounded the time to form the singularity by the initial data - no dimensional constants enter that might be used to tune away the singularity. It is worth emphasizing that if we were considering a gauge theory where $A_{\mu}$ were a gauge potential, then such caustic singularities would not be a concern. One could simply gauge them away. However, the Lagrange multiplier and coupling of the vector to the matter here mean there is no such gauge symmetry, and a singularity of the type here is a true physical singularity. The classical evolution of the theory is ill-posed once the singularity has formed.

In a sense we are done. Whilst in the absence of matter for more general initial data we have no argument to show caustics form, it certainly seems likely. This fact is already sufficient to render the dynamics of the TeVeS theory rather dubious. However since phenomenologically it is the interaction with matter that is of tantamount interest in TeVeS it is still interesting to understand whether matter ameliorates the situation or instead makes it worse and we spend the remainder of the paper investigating this.

An interesting and possibly related topic is the linear instability noted by Seifert [32] when considering perturbations of spherically symmetric backgrounds in Einstein-Æther and TeVeS using the methods of [33]. Clearly the phenomenon of caustic singularity formation is essentially non-linear and one would not expect to see it in linear theory. However the dynamics of such singularity formation may be associated with a growing unstable linear mode for the vector field, and it would be interesting to investigate whether there is indeed a link between our discussion and Seifert's linear instability. Seifert has discussed modifying TeVeS in the same sense we do later, and his work led to Skordis considering the same modification we present later in the context of cosmological perturbation theory.

\section{VECTOR FIELD DYNAMICS AND NEW STATIC BLACK HOLE GEOMETRIES}

In this section we find new classes of black holes in $\mathrm{TeVeS}$ where the Einstein metric is static, but the vector field has non-trivial dynamics, and its integral curves fall through the horizon. The previous static black hole geometries of Giannios [17] and of Sagi \& Bekenstein [24] have had the vector aligned with the killing time, which naively suggests the vector field in the exterior regions to slowly moving matter might dynamically wish to align with the matter's natural frame. However, the existence of our new solutions clearly shows that this is not to be expected, and that the dynamics of the vector exterior to a region containing matter may be very complicated, and in particular is likely to want to fall towards the matter and may form caustic singularities. For an actual matter source rather than a black hole where the integral curves cannot disappear through a horizon, accumulation of curves as the vector falls towards the matter are more likely to form singularities. Indeed in later sections our simulations with matter show that this is the case.

Later in the paper we will suggest a modification of the TeVeS theory to avoid caustic singularity formation. It is worth noting that the black hole solutions presented below will not be solutions in this modified theory and hence we avoid going into detailed phenomenology for these solution here. It would be interesting to study black hole solutions in the modified theory we suggest, and we make some comments on this in the concluding discussion section VI.

As discussed in the previous section we will be interested in length scales $L \ll \ell$, where the TeVeS scalar is approximately constant in the region of interest but varies enough to place the region in the strong acceleration regime. Thus, as discussed in Appendix A we may consider solutions to the TeVeS equations with exactly constant scalar, ignoring $\mathcal{F}$ and setting $\mu=1$ as a good approximation.

\section{A. New static black hole geometries with constant scalar}

It was shown by Giannios in [17] that if the vector field $A$ is aligned with the time translation Killing vector, then the solution for $\phi$ is singular unless it is constant. Letting that constant be $\phi_{c}$, the solutions take the form,

$$
\begin{aligned}
\phi & =\phi_{c} \\
A^{\mu} & =\left(\frac{1}{\sqrt{T(r)}}, 0,0,0\right), \\
d s^{2} & =-T(r) d t^{2}+R(r) d r^{2}+r^{2}\left(d \theta^{2}+\sin ^{2} \theta d \varphi^{2}\right),
\end{aligned}
$$

For our new solutions we again have a static Einstein metric and constant scalar, but now take $A^{r} \neq 0$,

$$
A^{\mu}=\left(A^{t}(r), A^{r}(r), 0,0\right),
$$

The scalar field equation is trivially satisfied for a vacuum spacetime. The vector field equation becomes

$$
K \nabla_{\beta} F^{\beta \alpha}+\lambda A^{\alpha}=0 . .
$$

For the case where $\alpha=r$ the first term vanishes leaving

$$
\lambda A^{r}=0,
$$


and thus for $A^{r} \neq 0$ we have $\lambda=0$. In this case the field equations become those of Einstein-Maxwell theory for a particular choice of gauge. Given this we expect to find
Reissner-Nordström (RN) black holes, and one can check this is indeed the general solution - we give the argument in appendix $\mathrm{B}$ 63. We find,

$$
\begin{aligned}
g & =\operatorname{Diag}\left[-\left(1-\frac{2 M}{r}+\frac{Q^{2}}{r^{2}}\right),\left(1-\frac{2 M}{r}+\frac{Q^{2}}{r^{2}}\right)^{-1}, r^{2}, r^{2} \sin ^{2}(\theta)\right], \\
A_{\mu} & =\partial_{\mu} \Phi+\delta_{\mu}^{t} \sqrt{\frac{2}{K}} \frac{Q}{r}, \\
\Phi & =-t \pm \int d r\left(1-\frac{2 M}{r}+\frac{Q^{2}}{r^{2}}\right)^{-1} \sqrt{\left(\frac{2}{K}-1\right) \frac{Q^{2}}{r^{2}}+\left(M+\sqrt{\frac{2}{K}} Q\right) \frac{2}{r}},
\end{aligned}
$$

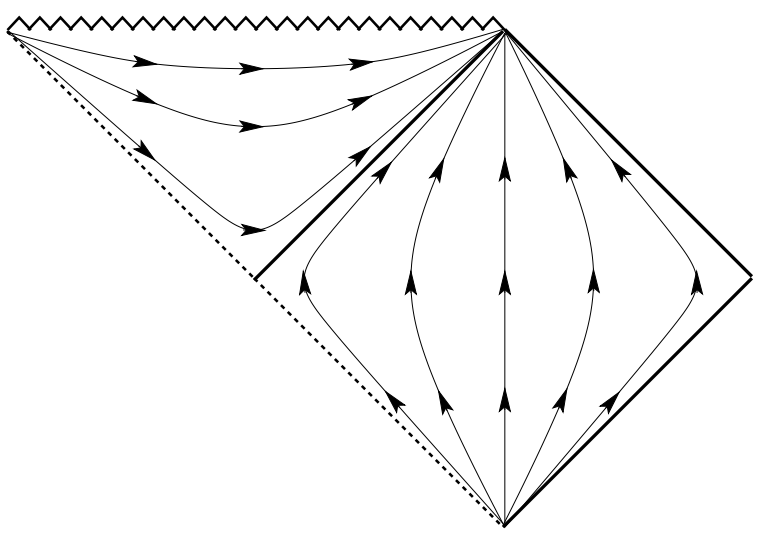

FIG. 3: Penrose Diagram for the Schwarzschild MF Spacetime where the vector field is aligned with the Killing time as in the Giannios solutions [17].

and hence see that the solution is indeed simply RN where the gauge freedom for the field $A$, specified by the function $\Phi$, has been fixed up to a sign by the Lagrange multiplier constraint that $A$ has unit timelike norm. Note that for $Q=0$ this gauge transformation $\Phi$ is the Lemaître time coordinate, i.e. the coordinate time experienced by in-falling geodesic observers. Clearly, $F_{\mu \nu}=0$ when $Q=0$ and so the integral curves of $A$ are geodesics of the EF geometry.

One may show that the matter frame metric is $\mathrm{RN}$ too, after an appropriate coordinate transformation. We cast $\tilde{g}$ into Schwarzschild-type coordinates, $(\tau, \rho)$ defined as,

$$
t(\tau, \rho)=e^{-\phi_{c}} \tau+f(\rho) \quad r(\rho)=e^{\phi_{c}} \rho,
$$

where $f$ is given by,

$$
f_{, \rho}=-e^{\phi_{c}} \frac{\tilde{g}_{r t}}{\tilde{g}_{t t}} .
$$

These transformations leave all components $\tau$ independent, and $\tilde{g}$ in standard RN form in Schwarzschild coor- dinates

$$
\tilde{g}_{\tau \tau}=-\frac{1}{\tilde{g}_{\rho \rho}}=-\left(1-\frac{2 \tilde{M}}{\rho}+\frac{\tilde{Q}^{2}}{\rho^{2}}\right) \quad \tilde{g}_{\tau \rho}=0,
$$

where now the mass and charge are given as,

$$
\begin{aligned}
e^{\phi_{c}} \tilde{M} & =e^{-4 \phi_{c}} M-\left(1-e^{-4 \phi_{c}}\right) \sqrt{\frac{2}{K}} Q, \\
e^{2 \phi_{c}} \tilde{Q}^{2} & =\left(e^{-4 \phi_{c}}+\left(1-e^{-4 \phi_{c}}\right) \frac{2}{K}\right) Q^{2} .
\end{aligned}
$$

This shows the metric is still RN, but with shifted horizon positions. For example, in the Schwarzschild case $(Q=$ 0 ) we have that the MF and EF horizons are related by

$$
r_{\mathcal{H}(\tilde{g})}=e^{-4 \phi_{c}} r_{\mathcal{H}(g)} \text {. }
$$

Bekenstein [8] demonstrates the speed of scalar perturbations (at fixed background vector) and vector perturbations (at fixed background scalar) is sub-luminal with respect to electromagnetic propagation only if $\phi>0 \mathrm{ev-}$ erywhere. So we conclude that in this case, the horizon seen by the matter fields is smaller than the horizon for the gravitational fields $(\phi, A, g)$.

We now approach TeVeS solutions from another direction. Starting with the standard RN solution in EinsteinMaxwell theory, we may obtain a vacuum solution of TeVeS with constant scalar and $\lambda=0$ provided we may choose a gauge such that the vector potential satisfies the TeVeS constraint $A^{\mu} A_{\mu}=-1$. Hence we find a large class of solutions with metric (32) and vector of the form (33) but where, as in the earlier equation (18), we regard the gauge condition as a first order p.d.e. in time for $\Phi$,

$$
\begin{aligned}
\partial_{t} \Phi= & -\sqrt{\frac{2}{K}} \frac{Q}{r}+\frac{1}{\left(-g^{t t}\right)}\left(g^{t i} \partial_{i} \Phi-\right. \\
& \left.\sqrt{\left(-g^{t t}\right)\left(1+\partial^{i} \Phi \partial_{i} \Phi\right)+\left(g^{t i} \partial_{i} \Phi\right)^{2}}\right) .
\end{aligned}
$$

Hence we may take the solutions to be characterized by the charges $M, Q$ and also $\Phi(t=0)$, from which we evolve 


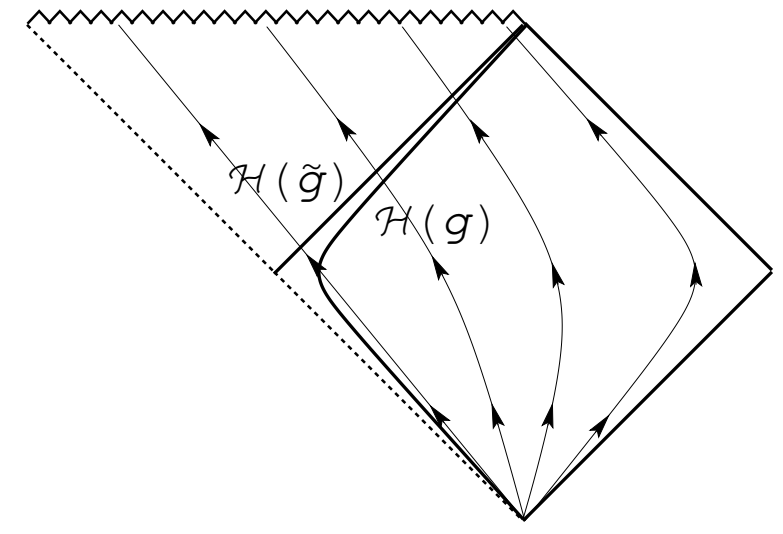

FIG. 4: The Penrose Diagram for the generalized Schwarzschild MF Spacetime $(Q=0$ case) with the EF horizon position. In this case the vector field is free-falling along geodesics of $g$ through both horizons.

to construct $\Phi$ for all $t$. The solution above in equation (34) is a stationary solution to this p.d.e. for $\Phi$. In general however the solutions to this p.d.e. will have complicated time dependence.

Note that for $Q=0$, this class of solutions has $R_{\mu \nu}=$ $F_{\mu \nu}=0$ as well as constant scalar and hence falls into our earlier class discussed in section Thus in this black hole background we can again precisely characterize caustic singularity formation by the previous statement that, letting $(\nabla \cdot A)_{0}$ be the value of $\nabla \cdot A$ on a surface $\Sigma$, then if $(\nabla \cdot A)_{0}<0$ at any point on $\Sigma$ a singularity will form within a proper time $-3(\nabla \cdot A)_{0}^{-1}$ measured along the future of the integral curve of $A$ through that point. Since this condition is generic within our class of solutions for $Q=0$, we see that caustic singularity formation is to be expected in the exterior of these black holes. Whilst we have no argument that the same is true for $Q \neq 0$, we expect it is likely.

The presence of the matter appears to attract the vector field integral curves. Naively this focusing would seemingly make caustic singularity formation more likely. However, interestingly the presence of the horizon actually renders the singularity formation less severe in the sense that if the time to singularity formation is sufficient that the integral curve of $A$ has fallen inside the horizon, an external observer need not care. Indeed in the stationary solution (34) above this precisely happens, with a caustic singularity occurring at the black hole singularity itself. Of course, if the matter source is not a black hole, but rather a compact object without horizon, then we still expect the vector field curve attraction, but now there is nowhere for the curves to go, and hence the expectation that the matter focuses the vector to form singularities would hold. Later in the paper we investigate this.

We conclude this section by commenting that we have examined the case of constant scalar, stationary black hole solutions. One might wonder whether stationary solutions with non-constant scalar can be found, which share the symmetry and asymptotics of those found here. We address this questions in the Appendix Cl finding evidence that no solutions exist near to the ones above with constant scalar. We show that for a linear perturbation of the scalar about our constant scalar solution a singular develops exterior to the horizon, and that performing a full non-linear numerical evaluation of such a solution one finds both the EF and MF metrics are nakedly singular. We cannot argue that no non-constant scalar black holes exist [64] but do expect there are none that are qualitatively similar to the constant scalar solutions we have found. Note that this is compatable with the argument presented in Appendix $\mathrm{A}$ since the kind of non-trivial scalar considered there need not be static nor share the same asymptotics or symmetry.

\section{VECTOR FIELD DYNAMICS AND MATTER: NUMERICAL SIMULATION}

In section II we have shown that in the absence of matter, a large class of deformations of the TeVeS vacuum initial data quickly terminate in caustic singularities upon time evolution. In section III we have shown that black hole solutions of $\mathrm{TeVeS}$ have complicated vector dynamics, which again include caustic singularities, and in particular that the black hole appears to attract the integral curves of the vector field toward it. We might then expect this to occur for any matter source, and then imagine that such an attraction which focuses the integral curves is likely to generate caustic singularities. This is too quick however, as matter couples to the vector field in $\mathrm{TeVeS}$ in a complicated fashion, and hence we have little intuition or analytic control over what happens.

It is the subject of this section to investigate the vector dynamics in the presence of matter using full numerical evolution of the TeVeS equations of motion. To make progress we restrict ourselves to spherical symmetry. We are then able to consider both gravitational collapse of a matter scalar field, and evolution of an initially near static boson star. In both cases we find the vector curves in the region exterior to the matter are indeed attracted towards the matter and do form caustic singularities. One might be concerned that imposing spherical symmetry restricts to a rather special class of solutions which focuses energy at the origin of spherical symmetry. However the caustic singularities actually form away from the origin and hence the singularities themselves locally have a planar symmetry, and seem not to result from the peculiarities of spherical symmetry. In both cases the TeVeS scalar is fully dynamical and non-constant, hence justifying the Newtonian regime approximation $(\mu=1$, neglecting $\mathcal{F}$ ). It remains smooth where the caustics form indicating that the scalar plays no role in the pathalogical vector dynamics.

Full details of the numerical implementation and convergence and constraint tests are postponed to Appen- 
dices $\mathrm{D}$ and $\mathrm{E}$.

\section{A. Scalar field collapse}

Our first matter system is the collapse of a complex scalar field. We perform an integration of the field equations from an initial spherical shell of scalar matter. We use a canonical complex scalar field $\chi$, whose action is constructed using the matter frame metric $\tilde{g}$. Using time symmetric initial data and a radial gaussian shell for the matter field $\chi$, the matter energy density will split into ingoing and outgoing components and for sufficient amplitude of the initial shell, the ingoing component will be focused at the origin into a high enough energy density to form a black hole. We use the coordinate system

$$
d s^{2}=-T^{2}(t, r) d t^{2}+e^{R(t, r)} d r^{2}+r^{2} d \Omega_{2}^{2} .
$$

which clearly covers only the exterior region of any black hole that might form. We emphasize that since the matter $\chi$ couples to the TeVeS vector and scalar, both must be evolved and have non-trivial dynamics.

For the metric we choose time symmetric initial data that satisfies the constraint equations. The TeVeS scalar we take to be constant initially, and time symmetric, and for the vector, we take $A^{r}=0$ at $t=0$ and choose $\dot{A}^{r}$ such that $F_{\mu \nu}=0$ at $t=0$. However, since there is matter, in contrast to the discussion in the previous sections above, $F_{\mu \nu}$ will immediately evolve to be nonzero, and $\phi$ to be non-constant.

We find that for different initial Gaussian shells of $\chi$, of sufficient amplitude to ensure non-linear dynamics when the ingoing pulse reaches the origin (for weak amplitudes the energy density simply passes through the origin and radiates to infinity as is the case in usual standard gravity coupled to a scalar) rather similar qualitative behaviour results. Figures 5, 6] and 7]give the results of a representative evolution. Even though $F_{\mu \nu} \neq 0$, we do see that the vector field curves are initially attracted to the matter shell. Evolution proceeds with the ingoing pulse deforming the geometry as for a usual scalar collapse. However before a horizon can form - recall our coordinate system only covers the black hole exterior - we see the formation of a caustic singularity. This is signaled by the divergence of $A$ which develops a growing spike on constant $t$ slices as seen in figure 6. At this point, dynamical evolution is no longer well defined. As noted above, the singularity forms well away from the origin of spherical symmetry. Figure 7 shows that the TeVeS scalar remains small and smooth up to this point, which suggests that dynamically it does very little, if anything, to prevent singularity formation. It is interesting that whilst we have found many candidate black hole end states for such a collapse in the previous section, the actual dynamics of the collapse is sufficiently badly behaved that we cannot even see an apparent horizon form.

One might worry that the choice of initial data with $F_{\mu \nu}=0$ is somewhat special (even though $F_{\mu \nu}$ evolves to be non-zero). [65] Indeed, since our earlier analytic arguments were for $F_{\mu \nu}=0$ it is useful to check that caustics may also form for initial data with $F_{\mu \nu} \neq 0$. Another question is whether the magnitude of $K, \kappa$ play a role in the singularity formation. For these reasons we present the result of another simulation in figures 8 , 9 and 10. These simulations used initial data for the vector where $\dot{A}_{r}=A_{r}=0$. Hence the initial data as a whole is now time symmetric, and $F_{\mu \nu} \neq 0$ initially. For the simulation shown we have also taken larger $K, \kappa$. We observe caustic formation again. Indeed the singularity forms earlier. Experimentally we find that for larger $K, \kappa$ a caustic forms earlier, which is to be expected as the vector is more strongly coupled to the dynamics of the other fields. Thus we see that for two very different choices of the vector initial data caustic singularities result.

\section{B. Perturbations to a boson star}

Scalar field collapse is an extreme dynamical process which is highly relativistic. It is interesting to consider whether an initially non-relativistic matter source also seeds an attraction of vector curves and subsequent caustic singularity. To this end we examine the full dynamics of $\mathrm{TeVeS}$ in the presence of an initially quasi-static boson star [34].

We use an identical numerical method and boundary conditions to integrate the field equations as for the scalar collapse above. To create the boson star we follow Gleiser [35]; as a matter source we use a complex scalar, $\chi$, now with potential $V(|\chi|)$. We begin by finding a static boson star solution. This is achieved by imposing the following separable solution to the $\chi$ equation of motion with the potential $V(|\chi|)=m^{2} \bar{\chi} \chi$

$$
\chi(t, r)=\chi_{0}(r) e^{\imath \omega t},
$$

where $\chi_{0}$ is real. While we term this a 'static' star, we note that in fact $\chi$ has the above phase rotation, although all other fields are indeed static. The metric functions $T, R$ are taken to depend only on $r$, and likewise for the TeVeS scalar. The TeVeS vector is chosen to be aligned with Killing time, so $A_{\mu}=(-T(r), 0,0,0)$. The radial profile for each of these functions is obtained via a shooting method - we fix the value of $\chi_{0}(0)$ and $m$ so that the resulting solution will have flattened out well before the boundary of our numerical grid. We then fine tune the value of $\omega$ to obtain the profile for $\chi_{0}$ with no nodes; this is the ground state star. We also choose the parameters so that the start has a low density compared to its radius and hence $T(0, r) \simeq 1$, so the backreaction of the star is weak - it is non-relativistic. Note that for this static star $F_{\mu \nu} \neq 0$, and the TeVeS scalar is non-constant.

To consider a dynamical perturbation of this static star we take similar initial data to the scalar collapse. We take $T, R$, the TeVeS scalar and the matter scalar to have initial data simply given by that on a constant time slice of the static boson star solution above. However we now 


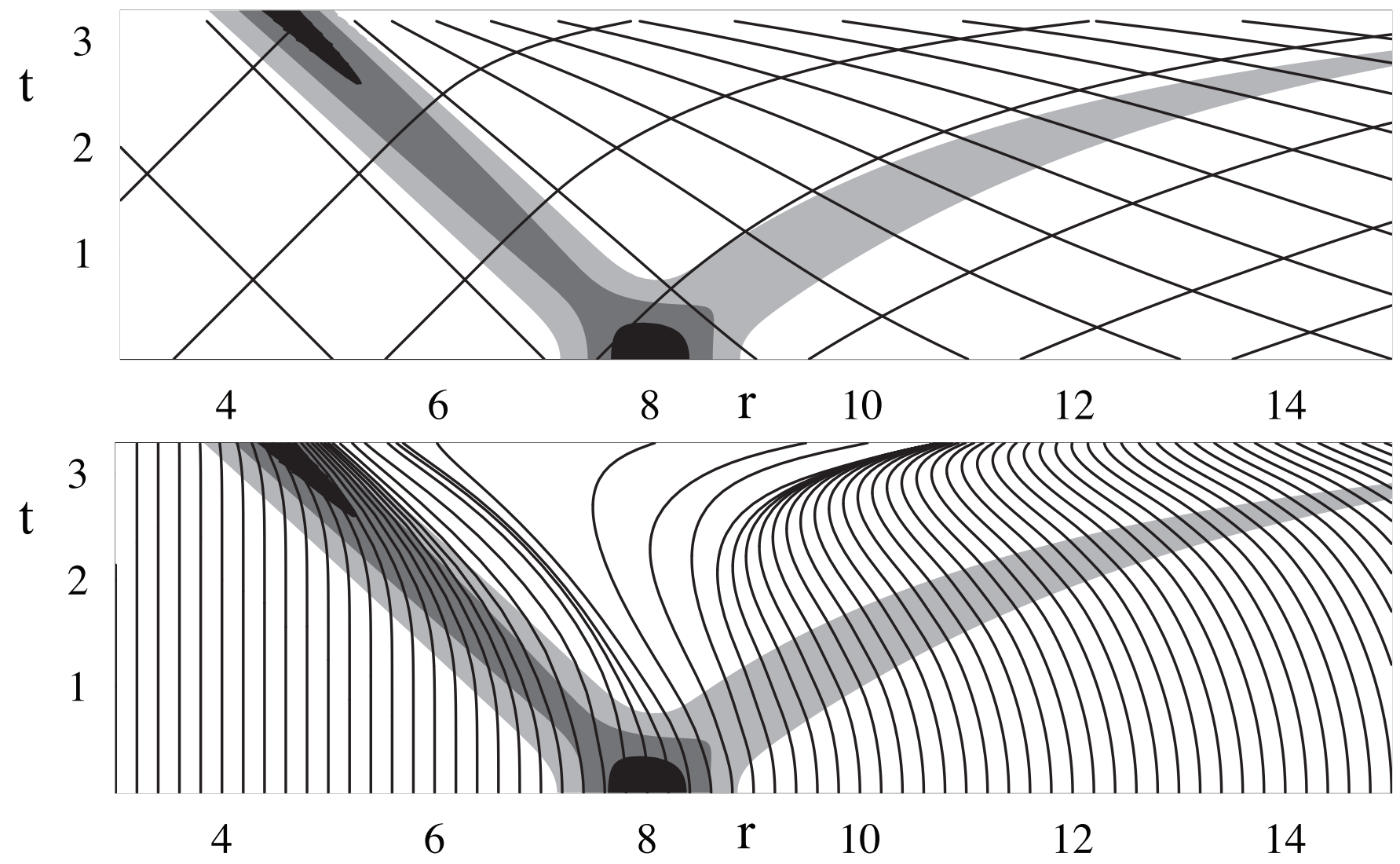

FIG. 5: Massless scalar field shell-collapse in an attempt to form a black hole. Upper Frame: The light cone structure is shown overlaying contours for the amplitude of the $\chi$ matter field. Lower Frame: Integral curves for the TeVeS vector $A$. In this evolution we see a caustic singularity develop around $r \simeq 11$ at $t \simeq 3.2$. The light cone structure indicates an apparent horizon is likely to have formed if the evolution had not been terminated by the caustic. The initial data for the vector was $A_{r}=0$ with $\dot{A}_{r}$ chosen so $F_{\mu \nu}=0$. The coordinate system is (42), with parameters $K=\kappa=0.01$

take $A^{r}=0$ and $F_{\mu \nu}=0$ at $t=0$ (although again $F_{\mu \nu}$ will not evolve to remain zero due to the boson star matter). Thus the vector is not now aligned with Killing time and dynamics will ensue. Note however that since the stars considered are in the non-relativistic regime, this perturbation to the vector field initial data is small. Hence the evolution is a non-relativistic process in its early stages .

We performed evolutions for a variety of star configurations, obtaining qualitatively similar results. A representative choice is illustrated in figure [1, 12 and 13. This shows that for our quasi-static initial configuration, the vector field curves fall in towards the star and do evolve to form a caustic singularity. However the singularity does not form in the interior of the star as one might naively expect. This is essentially due to the vector coupling to the matter, which apparently leads to a repulsive effect as we see the integral curves are clearly repelled from the origin of the spherical spatial geometry. While $F_{\mu \nu}$ is not zero outside the star, as it is sourced by the stellar matter, and then the region where $F_{\mu \nu}$ is non-zero propagates outward, we see that it does not stop the vector curves falling towards the star and eventually 'colliding' with the curves that were 'bounced' out of the interior of the star. The singularity appears rather similar in nature to that in the case of the scalar collapse, and figure 12 clearly shows a growing vector divergence at a finite radius as we approach the caustic. Figure 13 shows that once again the TeVeS scalar remains small and smooth up to the singularity.

Thus we have seen in this section that even starting with initial data whose short term evolution is nonrelativistic, pathological vector behaviour may quickly follow. In particular we see visually that since the integral curves are, at least initially, following an approximate timelike geodesic, the timescale for this singularity formation is of order the gravitational in-fall time. Thus in a Newtonian, quasi-static regime such as the neighborhood of the Earth, one might expect caustic singularity formation to occur on the order of hours, and after that point classical evolution is ill defined. This poor dynamical behaviour is clearly a serious obstruction to considering TeVeS as a phenomenological theory of modified gravity. 


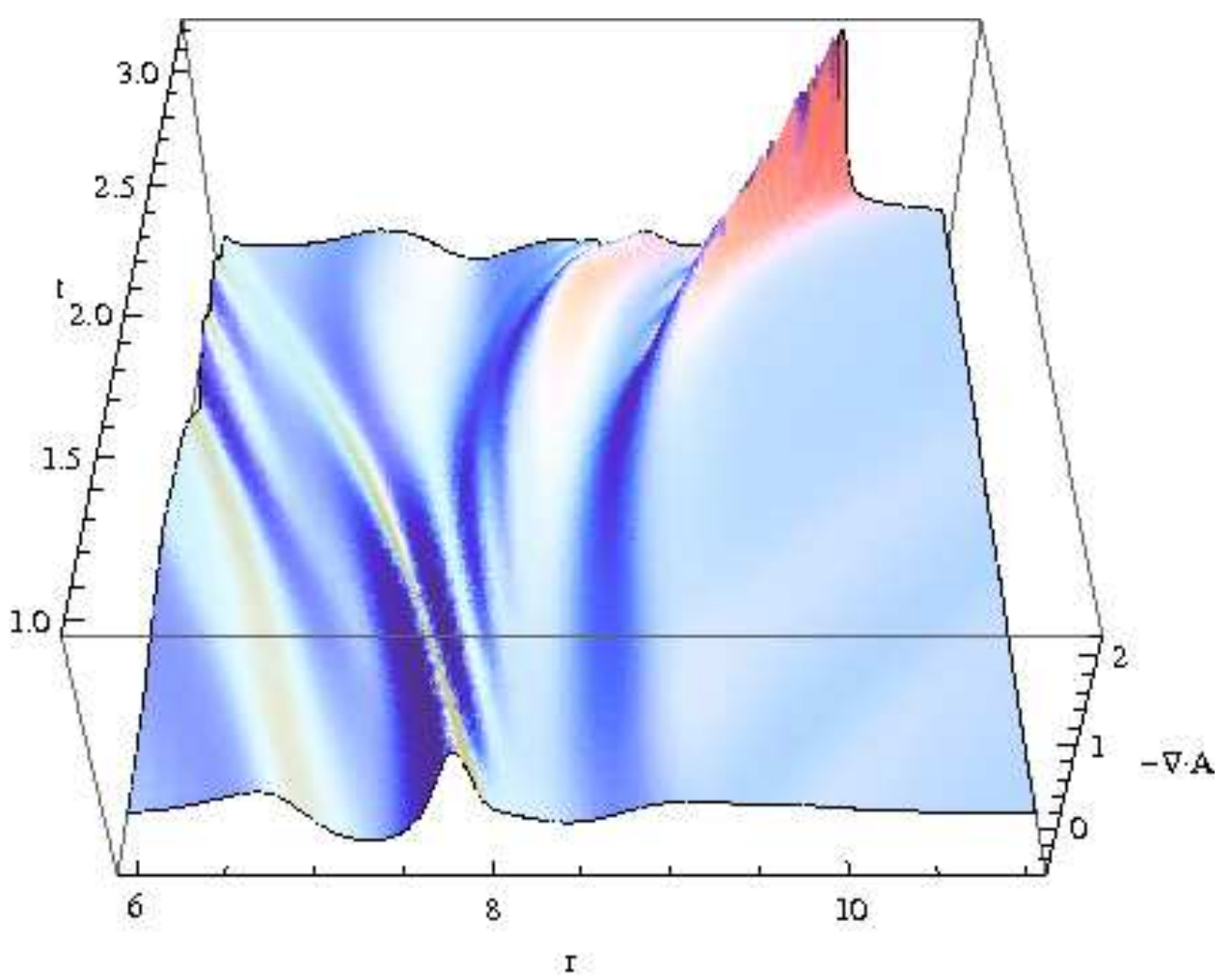

FIG. 6 : $-\nabla \cdot A$ for the simulation of massless scalar field shell-collapse, as in figure 5 Spiking of the 4-divergence of $-A$ corresponding to the convergence of its integral curves is easily seen, signalling the formation of a caustic.

\section{MODIFYING TEVES TO GET A WELL BEHAVED VECTOR DYNAMICS AND A MOND LIMIT}

Having demonstrated the formation of caustic singularities in $\mathrm{TeVeS}$ in various contexts which render the classical dynamics of the theory quickly ill defined, we now propose a correction to the vector part of the action (44) which may ameliorate this problem. The problem is essentially due to the Maxwell structure of the vector action. There is no energy cost when the divergence of the vector becomes large. Our modification is simply to introduce terms that disfavour large divergences. We simply take the vector action to be the most general diffeomorphism invariant action which is quadratic in derivatives and consistent with the $A^{2}=-1$ constraint. This action is of course precisely the one used for the vector in $\mathbb{E}$ theory. We begin with

$$
S_{g+v}=\frac{1}{16 \pi G} \int\left[R-\frac{K}{2} F_{\mu \nu} F^{\mu \nu}-\frac{c_{+}}{4} S_{\mu \nu} S^{\mu \nu}-c_{2}\left(\nabla_{\mu} A^{\mu}\right)^{2}-c_{4} \dot{A_{\mu}} \dot{A^{\mu}}+\lambda\left(A^{2}+1\right)\right](-g)^{1 / 2} d^{4} x,
$$

where $c_{+}=c_{1}+c_{3}, c_{1}-c_{3}=2 K, \dot{A}^{\mu}=A^{\nu} \nabla_{\nu} A^{\mu}$ and $S_{\mu \nu}=\nabla_{\mu} A_{\nu}+\nabla_{\nu} A_{\mu}$. We retain the pure TeVeS scalar action. The metric redefinitions which may performed in $\mathbb{E}$ theory [36] to remove one of these terms are no longer applicable here, as there are no non-trivial field redefinitions which leave the scalar action form-invariant at the same time as retaining the unit-norm constraint. As noted earlier, Seifert [32] has already proposed such a modification of TeVeS motivated by finding a linear insta- bility about certain spherically symmetric backgrounds (those of Giannios [17]) and leading from this Skordis [15] has recently derived the equations of motion and studied the cosmological perturbation equations. Our emphasis here is to check that the MoND limit is still recovered with this modification, and this has not previously been addressed - without this, of course, the modified TeVeS would be unlikely to provide any alternative explanations for dark matter. 


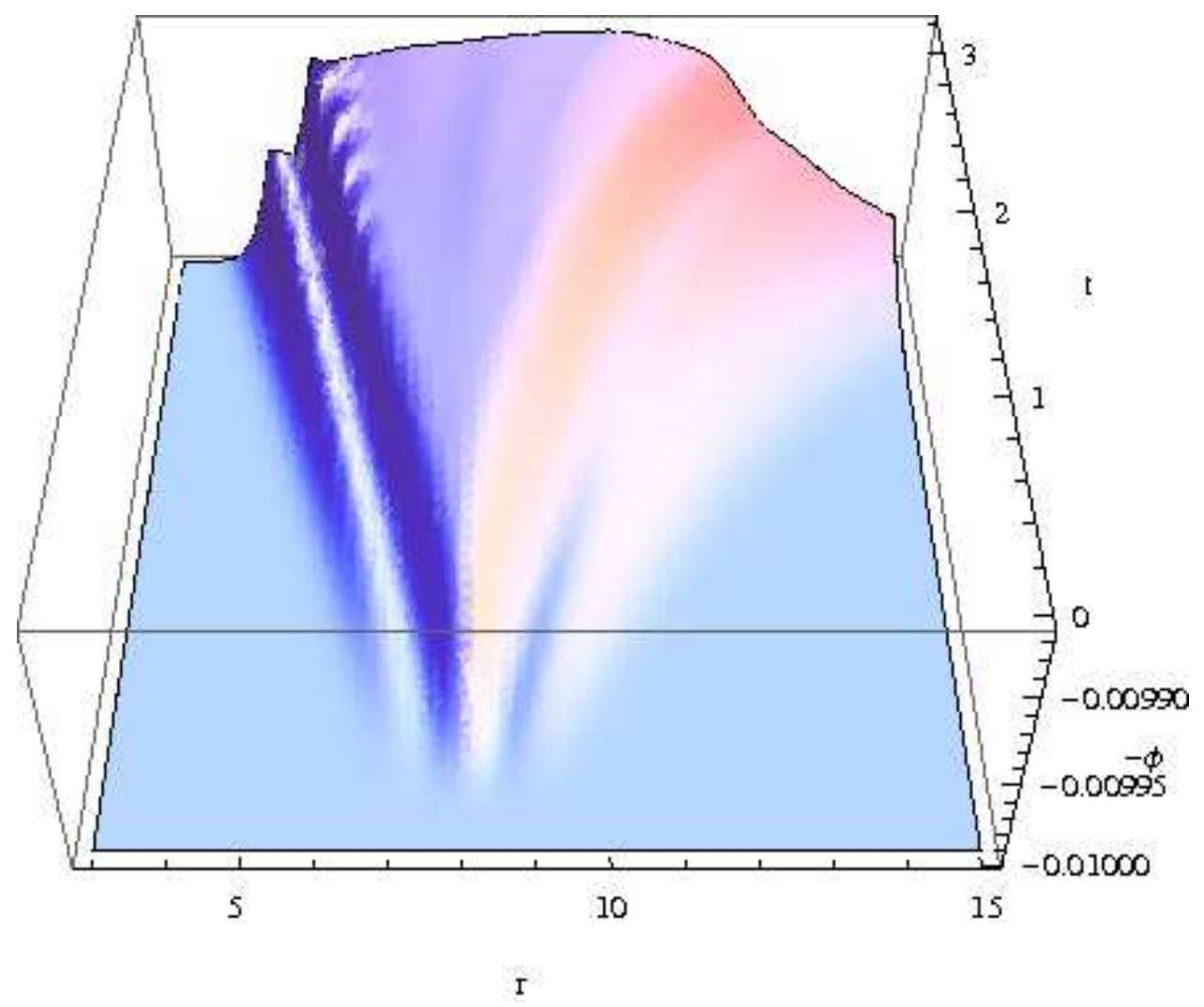

FIG. 7: $-\phi$ for the simulation of massless scalar field shell-collapse, as in figures [5] and 6] The scalar field remains small and smooth up to the formation of the caustic.

With this modified vector action, only the $\Theta$ term of the metric field equation is affected, and we obtain [66]

$$
\begin{array}{r}
\Theta_{\mu \nu}=K\left(F_{\sigma \mu} F_{\nu}^{\sigma}-\frac{1}{4} F^{2} g_{\mu \nu}\right)+\frac{c+}{2}\left(S_{\mu \sigma} S_{\nu}{ }^{\sigma}-\frac{1}{4} S^{2} g_{\mu \nu}+\nabla_{\sigma}\left[A^{\sigma} S_{\mu \nu}-S_{(\mu}^{\sigma} A_{\nu)}\right]\right) \\
+c_{2}\left(g_{\mu \nu} \nabla_{\sigma}\left(A^{\sigma} \nabla \cdot A\right)-A_{(\mu} \nabla_{\nu)} \nabla \cdot A-\frac{g_{\mu \nu}}{2}(\nabla \cdot A)^{2}\right) \\
+c_{4}\left(\dot{A}_{\nu} \dot{A}_{\mu}+\dot{A}_{\sigma} A_{(\mu} \nabla_{\nu)} A^{\sigma}-\nabla_{\sigma}\left[\dot{A}^{\sigma} A_{\mu} A_{\nu}\right]-\frac{g_{\mu \nu}}{2} \dot{A}_{\sigma} \dot{A}^{\sigma}\right)-\lambda A_{\mu} A_{\nu}, \\
K \nabla_{\mu} F^{\mu \nu}+\frac{c_{+}}{2} \nabla_{\mu} S^{\mu \nu}+c_{2} \nabla^{\nu}(\nabla \cdot A)-c_{4} \dot{A}^{\sigma} \nabla^{\nu} A_{\sigma}+c_{4} \nabla_{\sigma}\left(\dot{A}^{\nu} A^{\sigma}\right) \\
+\lambda A^{\nu}+8 \pi G \sigma^{2} A^{\mu} \phi_{, \mu} g^{\nu \gamma} \phi_{, \gamma}=8 \pi G\left(1-e^{-4 \phi}\right) g^{\nu \mu} \tilde{T}_{\mu \gamma} A^{\gamma},
\end{array}
$$

for the vector and scalar equations. We will refer to Bekenstein's theory as 'pure TeVeS', and the theory with the modification as 'modified TeVeS'.

The new parameters introduced into the action, $c_{+}, c_{2}, c_{4}$ will certainly be subject to physical constraints. The considerations are likely to be similar to those constraining the Einstein-Æther parameters, reviewed re- cently by Jacobson [31]. One complication is that the physical fluctuation modes of the vector now have different wave speeds when the more general vector action is introduced. In particular this leads to new constraints from Cherenkov radiation produced by cosmic rays [37, 38], although since the matter couplings are different from those of Einstein-Æther such analysis would 

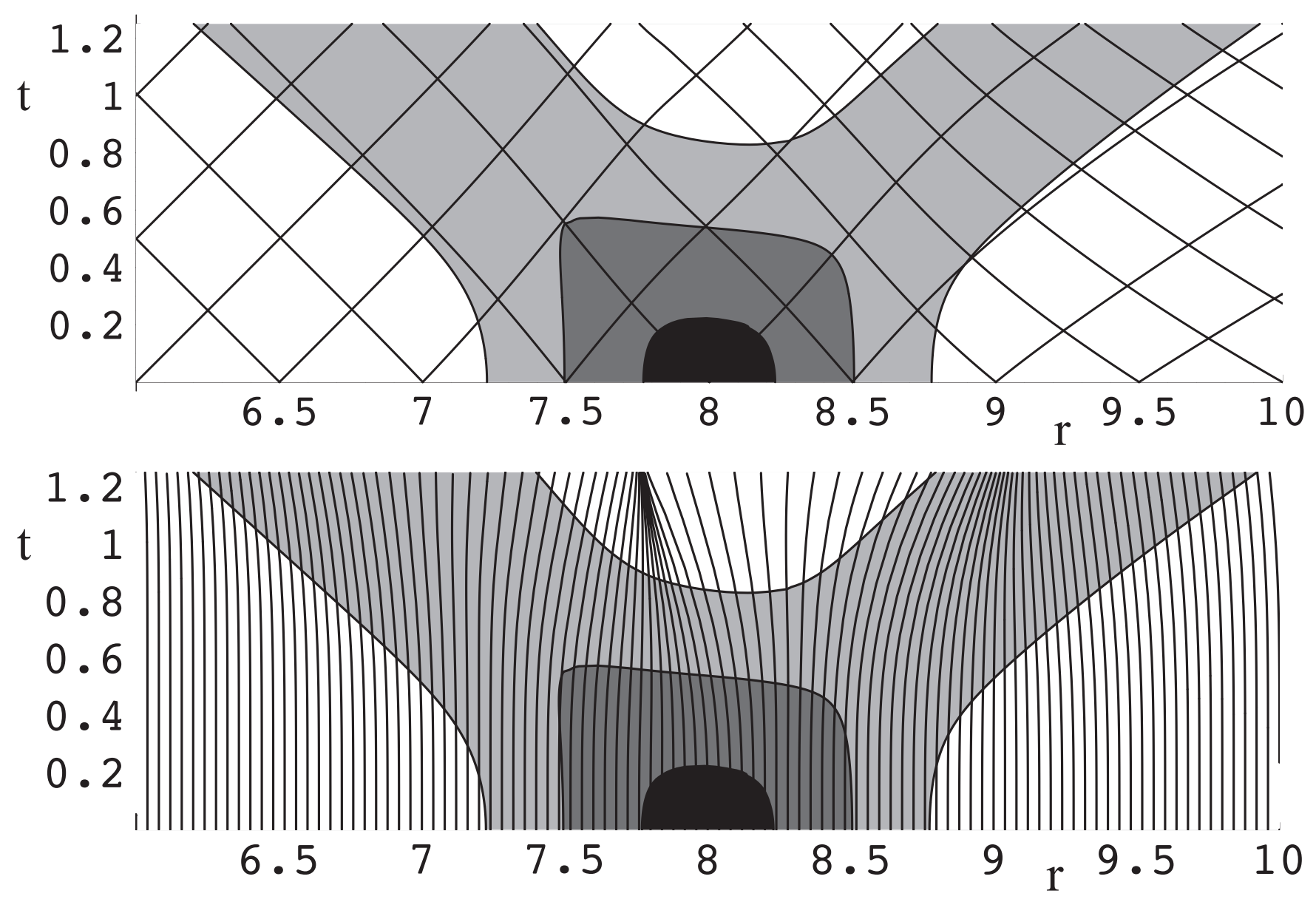

FIG. 8: As for figure 5] although now with initial data $\dot{A}_{r}=A_{r}=0$, so that $F_{\mu \nu} \neq 0$ initially. This simulation was performed with larger $K=\kappa=0.1$. We see again caustic formation, now much sooner.

likely have to be repeated for the modified TeVeS. We leave determination of constraints on these parameters for future work.

We have modified the TeVeS vector action in the hope that it will alleviate the problem of singularity formation. An absence of caustic formation is a crucial requirement for the theory to be dynamically well behaved, though we do not attempt to assess whether this is actually the case for our modified theory. We leave this as an interesting open problem. Now, assuming that the dynamics of this theory are in fact good, we would then require the theory to have the appropriate phenomenology. That is, we would like modified TeVeS to have inherited pure TeVeS's MoND limit. In order to check whether this is the case, we perform a Newtonian analysis where the goal is to obtain the equivalent of Poisson's equation for MF Newtonian potential. We perturb the EF metric to leading order in the Newtonian expansion as,

$$
g_{t t}=-1-2 V \quad g_{i j}=(1-2 V) \delta_{i j} \quad g_{t i}=0,
$$

where we linearize the equations in $V$, and ignore time derivatives at leading order. The matter source has only the non-trivial component $\tilde{T}_{t t}=\tilde{\rho}$. As for the EF metric, for the MF metric we take,

$$
\tilde{g}_{t t}=-1-2 \Phi \quad g_{i j}=(1-2 \Phi) \delta_{i j} \quad g_{t i}=0,
$$

and in the Newtonian expansion only the time component of $A$ is non-trivial at leading order, and is determined from $V$ by the condition $A^{\mu} A_{\mu}=-1$. The TeVeS scalar is written as $\phi=\phi_{c}+\delta \phi$. In the Newtonian limit at leading order we take $\delta \phi<<1$, and again neglect time derivatives. One may then verify that the disformal relation then relates these perturbations: $\Phi=V+\phi$.

Consider first the scalar field equation at leading order in the Newtonian expansion,

$$
\boldsymbol{\nabla} \cdot\left[\mu\left(\kappa l^{2}(\nabla \delta \phi)^{2}\right) \nabla \delta \phi\right]=\kappa G \tilde{\rho}
$$

Note that we have not linearised the argument of the $\mu$ function in $\delta \phi$ since while $\delta \phi<<1$, the TeVeS parameter $l$ is precisely large enough to balance this - hence we may recover MoND at leading order in the Newtonian analysis. There is no such subtlety in the other field equations, and we may straightforwardly perform a linearization in 


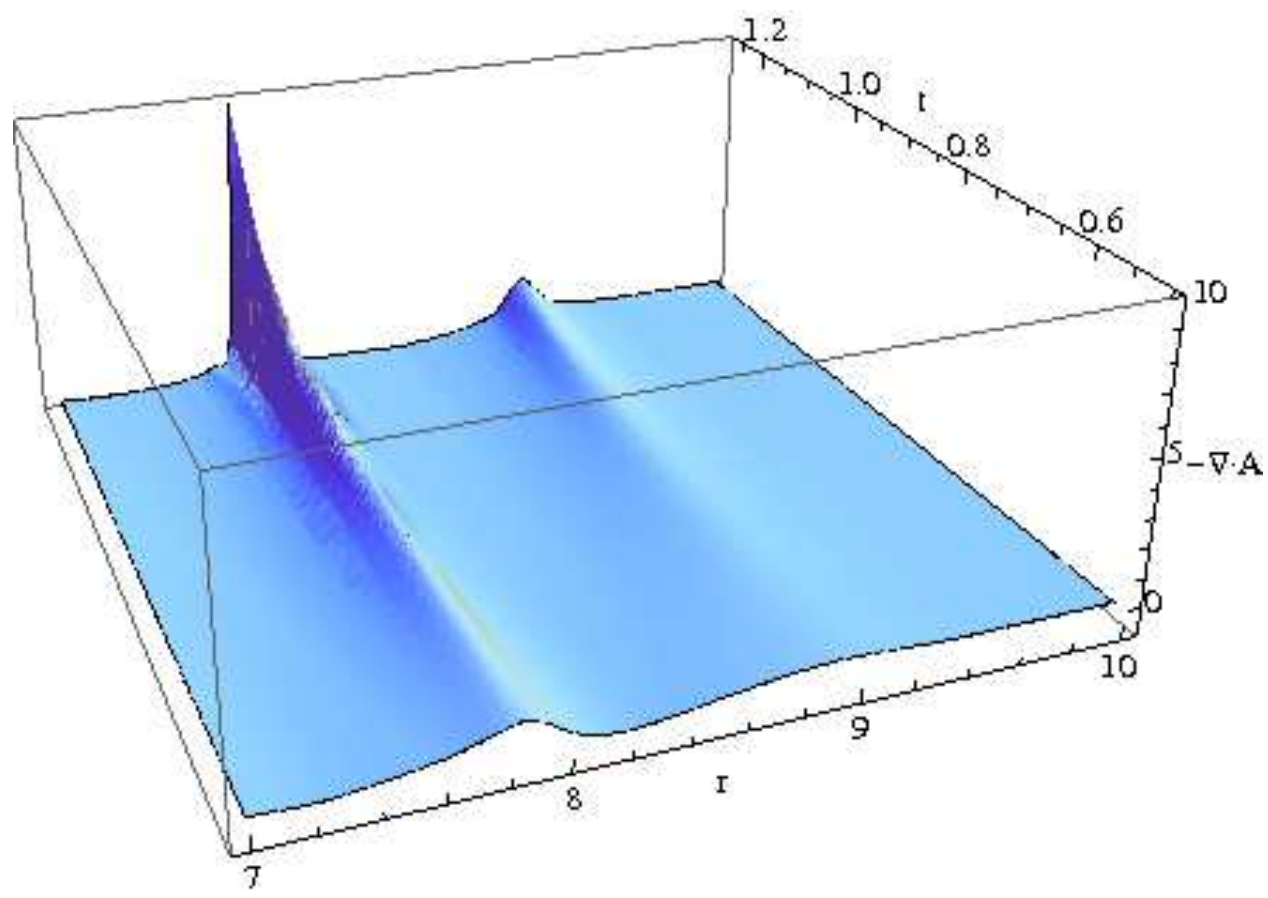

FIG. 9: $-\nabla \cdot A$ for the simulation in figure 8

the Newtonian potentials. All components of the vector field equation vanish except for the $t$-component,

$$
\lambda+\left(K-\frac{c_{+}}{2}\right) \nabla^{2} V=\left(-4 \phi_{c}\right) 8 \pi G \tilde{\rho},
$$

and similarly all components of Einstein's equation vanish with the exception of the $t t$-component,

$$
\lambda+\left(2+c_{4}-c_{+}\right) \nabla^{2} V=\left(1-8 \phi_{c}\right) 8 \pi G \tilde{\rho} .
$$

where we note that, as shown by Bekenstein [8], the term $\mathcal{F}$ doesn't contribute to the stress tensor in the leading order Newtonian limit. Combining the above results, we learn how $\Phi$ is related to $\Phi_{N}$ and $\phi$,

$$
\Phi=\left(1+\frac{2 K-2 c_{4}+c_{+}}{4}-4 \phi_{c}\right) \Phi_{N}+\phi .
$$

Hence, by following the same arguments that apply to pure TeVeS theory (as discussed by Bekenstein in [8]), MoND phenomenology results from (48) and (51) when $c_{4}$ and $c_{+}$are suitably constrained. Hence our proposed modification of TeVeS, which likely can avoid caustic formation for specific parameter ranges, does indeed correctly reproduce the MoND limit which is the raison d'etre for TeVeS.

\section{SUMMARY AND DISCUSSION}

We have argued that Bekenstein's original formulation of $\mathrm{TeVeS}$, while reproducing MoND phenomenology, is actually dynamically badly behaved. We have shown analytically and numerically in a variety of situations that the integral curves of the vector field quickly and generically evolve from regular initial data to caustic singularities. Once this occurs the classical evolution to the future is ill-posed.

Since the time scale to a singularity is not suppressed by any parameters, and is only determined by the initial data itself, it seems the original formulation of $\mathrm{TeVeS}$ is unlikely to provide a realistic theory of modified gravity. Put another way, while TeVeS does reproduce Newtonian and Modified Newtonian dynamics in a non-relativistic regime, it appears that in many cases this regime is unstable, the instability leading to the caustic singularities. We stress that already the analytic arguments of section III whilst made in the absence of matter, already highlight instability in the dynamics of TeVeS. The latter sections of the paper merely serve to illustrate that the situation remains unchanged when one considers the dynamics in the presence of matter. Indeed since matter can focus vector field curves towards it, it can make the situation worse.

It is useful to contrast this situation with the singularities that form in GR. We are very familiar with the fact that given certain initial data, matter can collapse to a singularity in GR, on the time scale of the gravitational in-fall time. However, in GR Cosmic Censorship means that these singularities are always hidden behind event horizons. Hence evolution outside the horizon is perfectly well defined. In contrast, the vector field singularities we have exhibited here lie outside any event horizon, and 


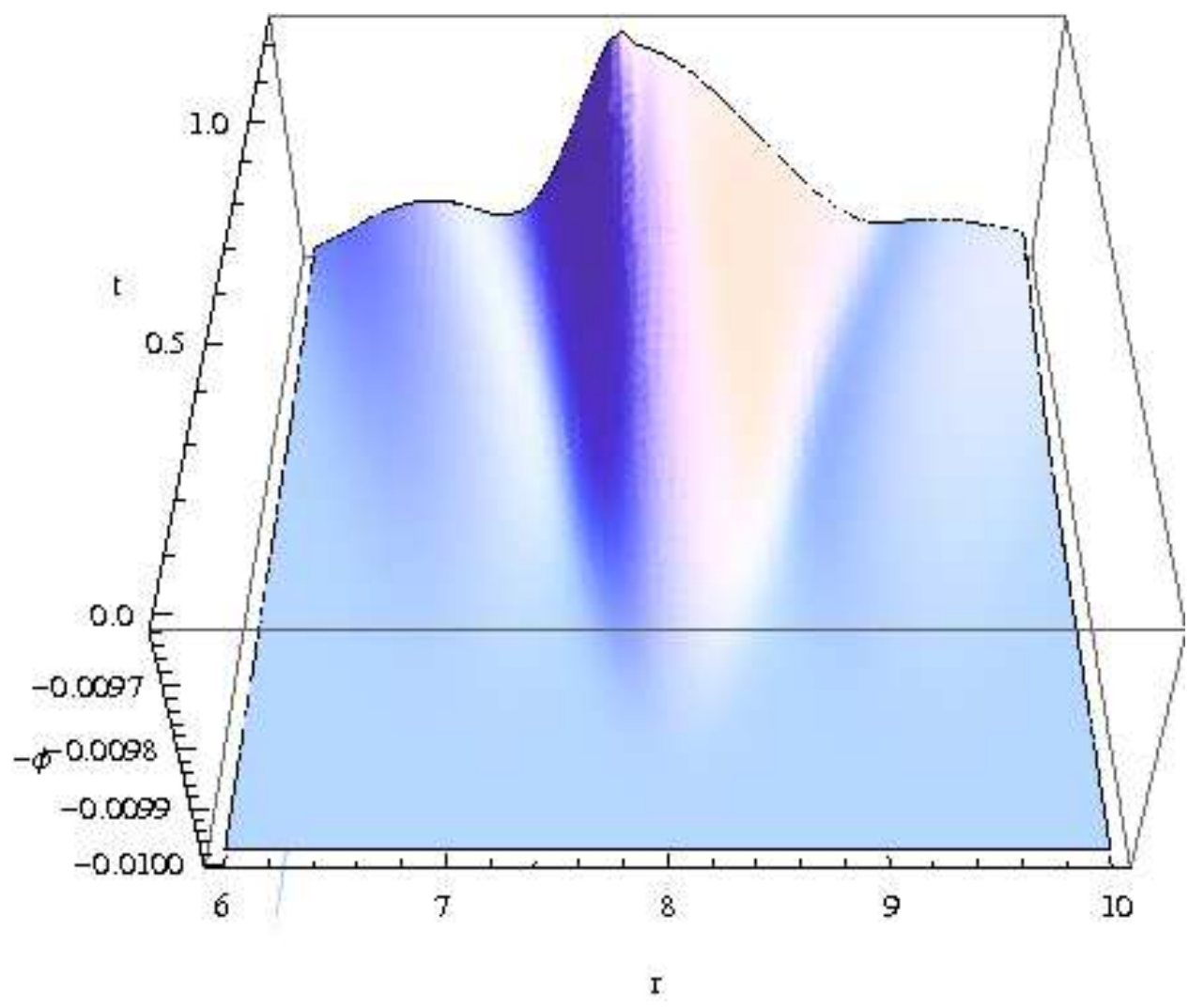

FIG. 10: $-\phi$ for the simulation in figures 8 and 9

hence evolution is impossible in the future null cone of these points unless there is some way to understand these singularities beyond the TeVeS effective field theory.

A similar situation arises in the perfect fluid, dust description of dark matter. The evolution of such a fluid also forms caustics (shocks) on time-scales of the in-fall time. Caustic formation in this case signals the breakdown of the fluid description of the dark matter and a requirement for a microscopic, particle description. Similarly our results indicate that the dynamical regime where $\mathrm{TeVeS}$ can be applied is limited by the breakdown of the effective theory on in-fall timescales. In this case however we don't have a microscopic description to transition to and the regime where the effective description appears to break down is relevant to the motivation of the theory itself.

Nonetheless, we believe a relatively minor modification, namely generalizing the vector action of TeVeS to a form like that of Einstein-Æther is likely to be able to give a dynamically well defined theory, which as we have shown still gives MoND behaviour for non-relativistic situations. It is interesting that a possibly related instability was observed for linear perturbations about the spherically symmetric static backgrounds of Giannios [17] by Seifert [32] and the same modification was proposed, although the recovery of the MoND limit had not previously been checked.
We emphasize that the detailed predictions of this modified TeVeS will likely differ from the original TeVeS theory, and therefore any phenomenological studies of $\mathrm{TeVeS}$ testing its ability to explain astrophysical or cosmological data without dark matter should be careful to include the necessary modification. It would be interesting to revisit the questions addressed in 12, 13, 18, 39, $40,41,42,43,44,45,46,47,48,49,50,51,52,53,54$, 55, [56, 57, 58] using the modified theory.

We have given large classes of new black hole solutions in the original TeVeS theory. However, these and the earlier solutions of Giannios are not solutions of the modified TeVeS theory. Instead in the case of modified TeVeS black hole solutions with a constant scalar (and it is likely there are not 'nearby' solutions with non-constant scalar) will be identical to those in Einstein-Æther theory discussed by Eling \& Jacobson [59, 60]. In particular there is no 'charge' parameter $Q$, with the static black hole geometries only being parametrized by one parameter, the mass $M$. However the qualitative behaviour of the solutions is somewhat similar to ours, with the integral curves of the vector field falling into the future horizon. It would be very interesting to study the geometry of the MF metric for these solutions. As a passing comment Eling et al. 61] have argued that such black holes may violate the Generalised Second Law. It is interesting that while the black hole solutions we found in the 


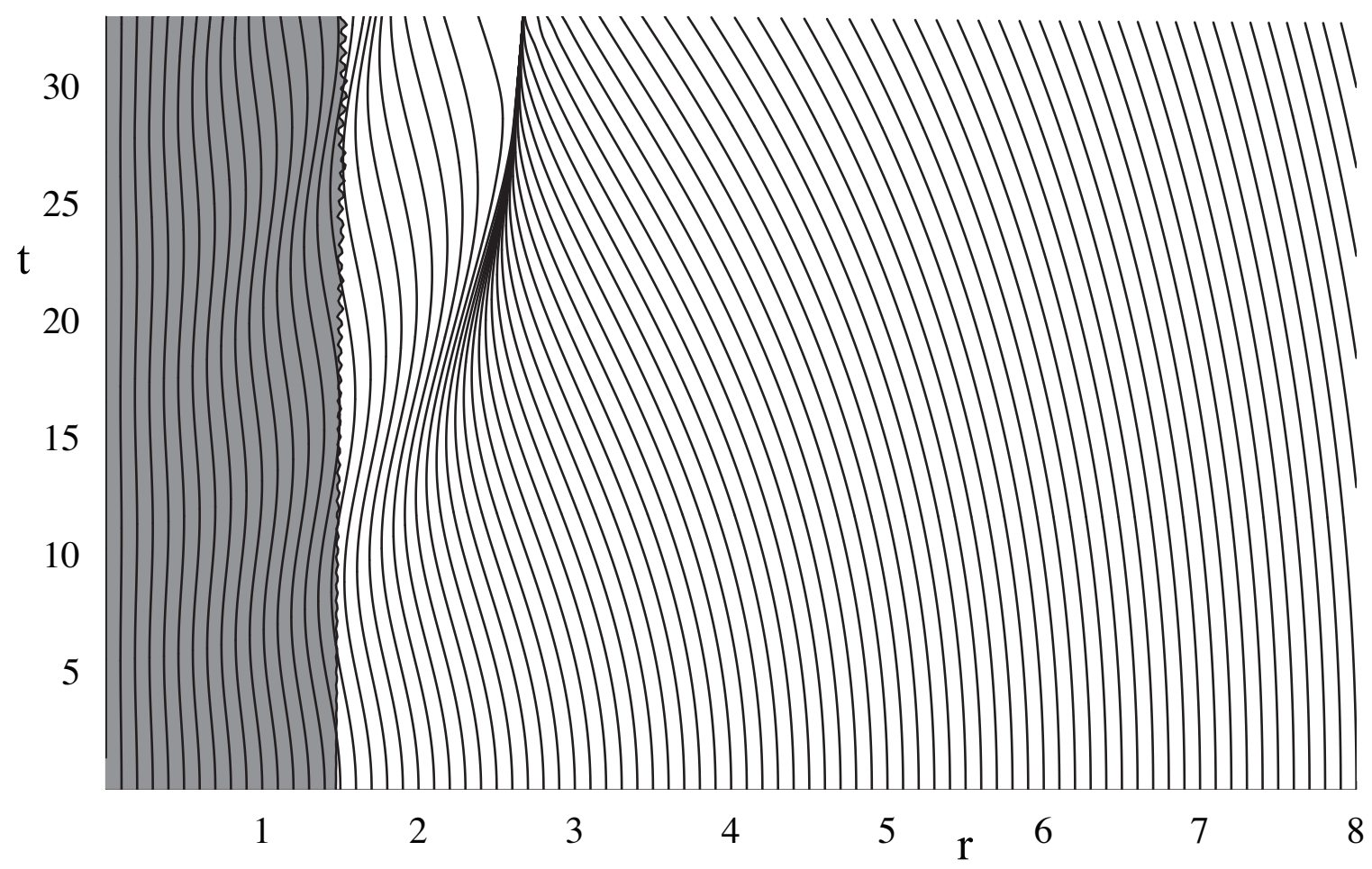

FIG. 11: A complex scalar field $\chi$ (grey shading) initially in the lowest static mode of a quadratic potential, as a matter source for a full TeVeS evolution. Shown also are the integral curves for the TeVeS vector $A$, illustrating the phenomenon of caustic formation where the initial data is quasi-static.

original TeVeS do not necessarily violate the law since their entropy depends on multiple charges $M$ and $Q$, for black hole solutions in the modified TeVeS there is only a one parameter family and the arguments of Eling et al. apply.

\section{Acknowledgements}

We wish to acknowledge Pedro Ferreira, Andrew Jaffe, and João Magueijo for useful discussions. We also thank Jacob Bekenstein and Martin Feix for valuable comments. TW is supported by an STFC advanced fellowship and a Halliday award. BW is supported by an STFC studentship. CRC acknowledges support from the Nuffield Foundation.

\section{APPENDIX A: APPROXIMATELY CONSTANT SCALAR SOLUTIONS IN THE NEWTONIAN REGIME}

In the main text we have made the claim that in the absence of matter we may formally consider solutions of $\mathrm{TeVeS}$ with constant scalar, and yet for the length scale of interest, let us call it $L$, which is much shorter than the TeVeS length scale $\ell$, we are still in the Newtonian regime, $\mu \simeq 1$. We envisage our scales of interest $L$ to be of order planetary or solar system scales. We claimed that to any solution with constant scalar, one can consider deforming the solution by adding scalar gradients that are tiny compared to our scale of interest $L$, and hence effectively negligible, but that would still be large enough over the region of interest to put the theory into the strong acceleration regime. The equation determining $\sigma$ is,

$$
-\mu \mathcal{F}(\mu)-\frac{1}{2} \mu^{2} \mathcal{F}^{\prime}(\mu)=y
$$

where we have written $\mu=\kappa G \sigma^{2}$ and $y=\kappa \ell^{2}|\phi|^{2}$ with $h^{\alpha \beta}=g^{\alpha \beta}-A^{\alpha} A^{\beta}$ and we have introduced the notation $|\phi|^{2} \equiv h^{\alpha \beta} \phi_{, \alpha} \phi_{, \beta}$. Let us also choose, following Bekenstein [8], an $\mathcal{F}$ such that in the Newtonian limit $\mu \rightarrow 1$, we have,

$$
\mathcal{F} \rightarrow \frac{3}{2} \ln (1-\mu), \quad y \rightarrow \frac{3}{4} \frac{1}{1-\mu}
$$

and hence in this limit we have,

$$
\mu=\kappa G \sigma^{2}=1+O\left(\kappa \ell^{2}|\phi|^{2}\right)^{-1} .
$$

We see a potential dilema in this claim is that naively $\mu \simeq 1, y \rightarrow \infty$ appears precisely at odds with a constant scalar which has $y=0$. Hence how can a constant scalar solution ever be 'close' to a solution in the strong acceleration regime $\mu \simeq 1$. The resolution is that what matters 


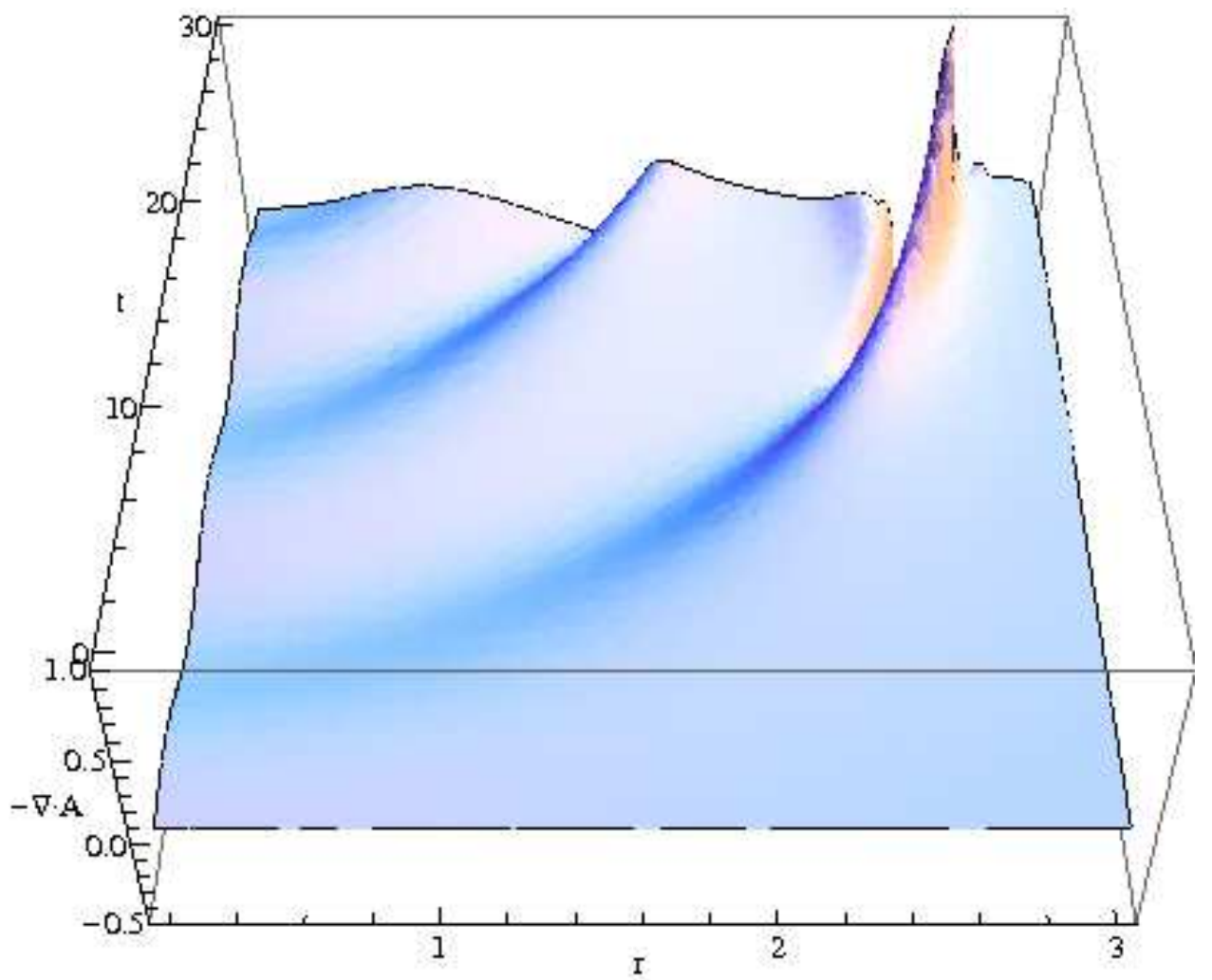

FIG. 12: $-\nabla \cdot A$ for the simulation of a complex scalar field as a ground state Boson star, as in figure 11 . Spiking of the 4-divergence of $-A$ corresponding to the convergence of its integral curves is easily seen, signalling the formation of a caustic singularity.

is not $|\phi|^{2}$, but $\ell^{2}|\phi|^{2}$, and hence one can have a tiny gradient on scales of interest $L$ over a region of size $L$, but provided $\ell$ is large enough, one can still have $\mu \simeq 1$. We will now formalize this more carefully, by providing a prescription to take a solution of Einstein-Maxwell theory and then generating a solution of TeVeS with almost constant scalar in a controlled manner.

In the absence of matter the scalar equation reduces to,

$$
\nabla_{\beta}\left(\mu\left(\kappa \ell^{2}|\phi|^{2}\right) h^{\alpha \beta} \phi_{, \alpha}\right)=0
$$

and the vector, which satisfyies the constraint $A^{\mu} A_{\mu}=$ -1 , obeys,

$$
K \nabla_{\beta} F^{\beta \alpha}+\lambda A^{\alpha}+\frac{8 \pi}{\kappa} A^{\beta} \phi_{, \beta} g^{\alpha \gamma} \phi_{, \gamma}=0,
$$

with the Einstein equations governing the metric becoming,

$$
G_{\alpha \beta}=\Theta_{\alpha \beta}^{\text {scalar }}+\Theta_{\alpha \beta}^{\text {vector }}+\Theta_{\alpha \beta}^{\mathcal{F}},
$$

with,

$$
\begin{array}{r}
\Theta_{\alpha \beta}^{\text {scalar }} \equiv 8 \pi G \sigma^{2}\left[\phi_{, \alpha} \phi_{, \beta}-\frac{1}{2} g^{\mu \nu} \phi_{, \mu} \phi_{, \nu} g_{\alpha \beta}-\right. \\
\left.A^{\mu} \phi_{, \mu}\left(A_{(\alpha} \phi_{, \beta)}-\frac{1}{2} A^{\nu} \phi_{, \nu} g_{\alpha \beta}\right)\right]
\end{array}
$$

$$
\begin{gathered}
\Theta_{\alpha \beta}^{\text {vector }} \equiv K\left(F_{\alpha}{ }^{\mu} F_{\beta \mu}-\frac{1}{4} g_{\alpha \beta} F^{2}\right)-\lambda A_{\alpha} A_{\beta} \\
\Theta_{\alpha \beta}^{\mathcal{F}} \equiv-2 \pi G^{2} \ell^{-2} \sigma^{4} \mathcal{F}\left(\kappa G \sigma^{2}\right) g_{\alpha \beta}
\end{gathered}
$$

We consider the dimensionless constants $K, \kappa$ to be small but order one in what follows.

Let us now consider a solution, $\hat{A}_{\alpha}, \hat{g}_{\alpha \beta}$ of EinsteinMaxwell theory with gauge constraint $\hat{A}^{\mu} \hat{A}_{\mu}=-1 \mathrm{im}-$ posed using the Lagrange multiplier $\lambda$ as for TeVeS,

$$
\begin{aligned}
& \hat{G}_{\alpha \beta}=K\left(\hat{F}_{\alpha}{ }^{\mu} \hat{F}_{\beta \mu}-\frac{1}{4} \hat{g}_{\alpha \beta} \hat{F}^{2}\right)-\lambda \hat{A}_{\alpha} \hat{A}_{\beta} \\
& K \hat{\nabla}_{\beta} \hat{F}^{\beta \alpha}+\hat{\lambda} \hat{A}^{\alpha}=0 .
\end{aligned}
$$

Here quantities with hats are composed from $\hat{A}_{\alpha}, \hat{g}_{\alpha \beta}$. Let us characterize the length scales of interest by the length $L$, and restrict our attention to a spacetime region $V$ of spatial size $\sim L$. Hence the curvatures of interest in the solution $\left(\hat{A}_{\alpha}, \hat{g}_{\alpha \beta}\right)$ will scale as $1 / L^{2}$ are we are not interested in much smaller curvature scales. We are envisaging that this scale is of order the solar system or less and compared to the TeVeS lengthscale $\ell$ we have a vast separation of scales,

$$
L<<\ell \text {. }
$$




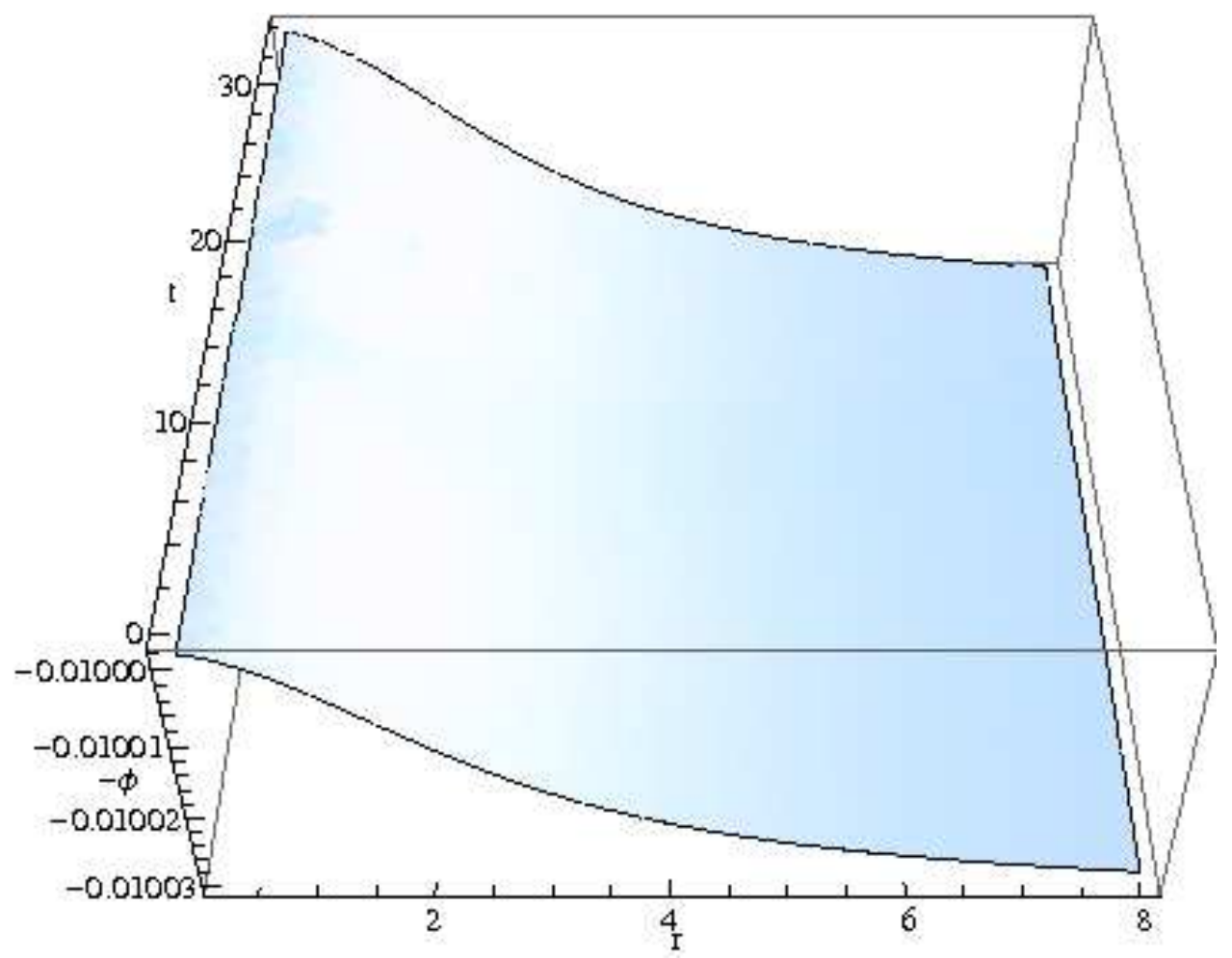

FIG. 13: $-\phi$ for the simulation of a complex scalar field as a ground state Boson star, as in figures 11 and 12 .

We begin by constructing a solution for a scalar $\hat{\phi}$ on the fixed solution $\left(\hat{A}_{\alpha}, \hat{g}_{\alpha \beta}\right)$ in the spacetime region $V$, of spatial scale $\sim L$. We take the scalar to obey the equation,

$$
\hat{\nabla}_{\beta}\left(\hat{h}^{\alpha \beta} \hat{\phi}_{, \alpha}\right)=0
$$

where $\hat{h}^{\alpha \beta}=\hat{g}^{\alpha \beta}-\hat{A}^{\alpha} \hat{A}^{\beta}$, and we emphasize that we are ignoring any backreaction - it is simply a scalar on our fixed solution. We require that in our region $V$ the solution everywhere obeys the condition,

$$
1>>L^{2} \hat{h}^{\alpha \beta} \hat{\phi}_{, \alpha} \hat{\phi}_{, \beta}>>\frac{L}{\ell} .
$$

For example, taking the trivial flat space solution $\hat{g}_{\alpha \beta}=$ $\eta_{\alpha \beta}, \hat{A}=\frac{\partial}{\partial t}$, one might choose the scalar to be $\hat{\phi}=\alpha x$ where $x$ is one of the spatial coordinates, and $\alpha$ is a constant in the range $1>>L^{2} \alpha^{2}>>L / \ell$. In general we expect to be able to find solutions obeying the condition (A13), although we do not have a formal existance proof of this.

From this Einstein-Maxwell solution and the associated scalar solution $\left(\hat{\phi}, \hat{A}_{\alpha}, \hat{g}_{\alpha \beta}\right)$ we may construct an approximate solution of the TeVeS equations, $\left(\phi, A_{\alpha}, g_{\alpha \beta}\right)$, perturbatively in the dimensionless constant,

$$
\epsilon=\left(\frac{L}{\ell}\right)^{1 / 4}
$$

as,

$$
\begin{aligned}
g_{\mu \nu} & =\hat{g}_{\mu \nu}+\epsilon g_{\mu \nu}^{(1)} \\
A_{\mu} & =\hat{A}_{\mu}+\epsilon A_{\mu}^{(1)} \\
\lambda & =\hat{\lambda}+\epsilon^{2} \lambda^{(1)} \\
\phi & =\phi_{0}+\epsilon \hat{\phi}+\epsilon^{2} \phi^{(1)}
\end{aligned}
$$

where $\phi_{0}$ is a constant and plays no role in the vacuum TeVeS equations which only involve $\phi$ derivatives. Taking the limit $\epsilon \rightarrow 0$, ie. looking at small scales compared to the TeVeS length scale $\ell$, we therefore see that the dynamics of TeVeS on scales $\sim L$ is given by precisely the Einstein-Maxwell solution $\left(\hat{A}_{\alpha}, \hat{g}_{\alpha \beta}\right)$ and hence by an effectively (although not precisely) constant TeVeS scalar. We may think of the $\epsilon \rightarrow 0$ limit as fixing $L$ and taking $\ell$ to infinity, or alternatively and more physically for fixed $\ell$, focussing our interest on smaller and smaller length scales $L$.

Let us now check this claim. Firstly let us consider the scalar equation at leading order in the $\epsilon$ expansion. Consider the behaviour of $\mu\left(\kappa l^{2}|\phi|^{2}\right)$. From our condition (A13) above we see that,

$$
1>>\frac{L^{2}}{\epsilon^{2}}\left(h^{\alpha \beta} \phi_{, \alpha} \phi_{, \beta}+O\left(\epsilon^{2}\right)\right)>>\frac{L}{\ell} .
$$

so that for $\epsilon \rightarrow 0$ we have,

$$
\ell^{2} h^{\alpha \beta} \phi_{, \alpha} \phi_{, \beta}>>\frac{1}{\epsilon^{2}}
$$


and thus we see that,

$$
\mu\left(\kappa l^{2}|\phi|^{2}\right) \simeq 1-O\left(\epsilon^{2}\right)
$$

so that in our region $V$ we are forced into the Newtonian regime of TeVeS, even though the scalar field gradient is perturbatively small compared to the scale $L$ of interest in our region. Hence the TeVeS scalar equation becomes,

$$
\nabla_{\beta}\left(h^{\alpha \beta} \phi_{, \alpha}\right)=O\left(\epsilon^{3}\right),
$$

with the right-hand side coming from the non-constant part of $\mu$. This is indeed consistent with our ansatz (A15) above for the constant and $\hat{\phi}$ pieces with the correction term, $\phi^{(1)}$, accounting for the lower orders. $\phi^{(1)}$ is sourced primarily by the $O(\epsilon)$ corrections to $\nabla_{\alpha}$ and $h^{\alpha \beta}$, from the metric and vector corrections $A_{\alpha}^{(1)}$ and $g_{\alpha \beta}^{(1)}$, with the source from $\mu$ actually being sub leading to this.

We now show that just as the scalar equation is consistently solved perturbatively in $\epsilon$ by our ansatz, the Einstein and vector equations are too. In particular we must show that the backreaction in the Einstein equations from the scalar $\phi$ and TeVeS function $\mathcal{F}$ are small compared to the characteristic curvature scale $1 / L^{2}$ in the solution $\left(\hat{g}_{\mu \nu}, \hat{A}_{\mu}\right)$. Now following from our condition (A13) we have that the scalar in our region obeys the bound,

$$
\frac{\epsilon^{2}}{L^{2}}>>h^{\alpha \beta} \phi_{, \alpha} \phi_{, \beta},
$$

and in addition we have an estimate for the contribution of $\mathcal{F}$ in the Einstein equations,

$$
\begin{aligned}
\Theta_{\alpha \beta}^{\mathcal{F}} & \sim \frac{1}{\ell^{2}} \ln (1-\mu) g_{\alpha \beta} \\
& \sim \frac{1}{L^{2}}\left(\epsilon^{8} \ln \epsilon\right) \hat{g}_{\alpha \beta} .
\end{aligned}
$$

Hence we see that the Einstein and vector equations to lowest order in $\epsilon$ reduce simply to the Einstein-Maxwell ones, and our ansatz (A15) therefore solves them to lowest order. The leading higher order corrections come from the perturbatively small backreaction of the scalar, and lead to the $O(\epsilon)$ corrections to $g_{\mu \nu}, A_{\mu}$, with the TeVeS function $\mathcal{F}$ essentially being negligible in the Newtonian limit as discussed in Bekenstein's original paper.

We have now more carefully justified our claim in the main text, namely that we may consider the TeVeS scalar to be effectively constant, and still be in the Newtonian regime $\mu \rightarrow 1$, provided we are restricting our interest to a region of scale $L<<l$, as we are in the main text. Our first application is to consider a bound on caustic formation time using Raychaudhuri's equation for the EinsteinMaxwell system. Since caustic formation is local, we are only concerned with the spacetime in the region of scale $L$ where the caustic forms, and not the asymptotic behaviour of our geometry. The physical setting would be caustic formation on, for example, solar system scales $L$, with the gradient of the scalar arising from much larger galactic scales $\ell$. The second application is to embed the Einstein-Maxwell black holes in TeVeS. Again, since we are not interested in the far asymptotic region of these solutions, we may again employ our approximation to ignore scalar gradients and the TeVeS function $\mathcal{F}$ in the stress energy. For a region of size $L$ surrounding the black hole, the corrections will be characteristic scale $\ell$ and for any astrophysical black hole, phenomenologically this region of interest certainly obeys $L<<\ell$.

We conclude with our previous example; $\hat{g}_{\alpha \beta}=\eta_{\alpha \beta}$, $\hat{A}=\frac{\partial}{\partial t}, \hat{\lambda}=0$, and scalar solution on this background $\hat{\phi}=\alpha x$ with the constant $\alpha$ obeying $1>>L^{2} \alpha^{2}>>$ $L / \ell$. Hence we may take $\alpha=\epsilon / L$. In this case the exact $\mathrm{TeVeS}$ solution can be found in the absence of the $\mathcal{F}$ term, which we have argued is subdominant over the other corrections;

$$
\begin{aligned}
d s^{2} & =-a(x)^{2} d t^{2}+d x^{2}+\frac{1}{a(x)}\left(d y^{2}+d z^{2}\right) \\
A & =\frac{1}{a(x)} \frac{\partial}{\partial t} \\
\phi & =\phi_{0}+\epsilon \frac{x}{L}
\end{aligned}
$$

with the function,

$$
a(x)=A e^{ \pm \epsilon \frac{4 \sqrt{\pi}}{\sqrt{\kappa(2 K-3)}} \frac{x}{L}}
$$

and one finds $\lambda=16 K \pi \alpha^{2} /(\kappa(2 K-3))$. Note that $y$ is actually a constant for this solution, $y=\kappa \epsilon^{-7 / 4}$, so that $\mu$ is also constant. Expanding in $\epsilon$ one finds this to be consistent with the ansatz. We have ignored the $\mathcal{F}$ term in this exact solution and a calculation confirms this is of order the estimate (A21), and hence vastly subdominant to the leading $\epsilon$ corrections in the solution.

\section{APPENDIX B: CONSTANT SCALAR STATIC BLACK HOLE DERIVATION}

We begin with a spherically symmetric, stationary system in Schwarzschild coordinates for which,

$$
\begin{aligned}
\phi= & \phi_{c}, \\
A^{\mu}= & \left(A^{t}(r), A^{r}(r), 0,0\right), \\
d s^{2}= & -T(r) d t^{2}+R(r) d r^{2}+ \\
& r^{2}\left(d \theta^{2}+\sin ^{2} \theta d \varphi^{2}\right),
\end{aligned}
$$

where $\phi_{c}$ is constant. The scalar field equation is trivially satisfied for a vacuum spacetime. The vector field equation becomes

$$
K \nabla_{\beta} F^{\beta \alpha}+\lambda A^{\alpha}=0 . .
$$

For the case where $\alpha=r$ in equation (B4), the first term vanishes, leaving the branch choice

$$
\lambda A^{r}=0,
$$


thus for $A^{r} \neq 0$ we have $\lambda=0$ everywhere. In this case the field equations become those of Einstein-Maxwell theory for a particular choice of gauge. Given this we expect to find Reissner-Nordström black holes. Expressing the field equations in terms of our metric and vector ansätze, we obtain three useful components of the Einstein equations, $(\theta \theta) \frac{1}{2 R T}\left(2 T^{\prime} R T-2 R^{\prime} T^{2}-T^{\prime} R^{\prime} T r+T^{\prime \prime} R T r-T^{2} R r\right)=K r\left(A_{t}^{\prime}\right)^{2}$, where primes indicate derivatives with respect to $r$. Eliminating $A_{t}^{\prime}$ between equations (B6) and (B7) leads to the relation

$$
T R=C_{1},
$$

where $C_{1}$ is a constant, which we set to 1 using the freedom available in rescaling the $t$ coordinate at this stage. Performing the same elimination between equations (B7) and (B8) and substituting for $T$ using (B9), we arrive at a solution for the second metric component,

$$
R=\left(1-\frac{2 M}{r}+\frac{Q^{2}}{r^{2}}\right)^{-1},
$$

with the corresponding solution for $T$,

$$
T=\left(1-\frac{2 M}{r}+\frac{Q^{2}}{r^{2}}\right)
$$

where $M$ and $Q^{2}$ are integration constants. The quantity $Q^{2}$ will indeed turn out to be positive.

Consider the $t$ component of the co-vector field equation

$$
\begin{array}{r}
\frac{1}{2 T R^{2} r}\left(2 K r A_{t}^{\prime \prime} R T-K r A_{t}^{\prime} T^{\prime} R-K r A_{t}^{\prime} R^{\prime} T+\right. \\
\left.4 K A_{t}^{\prime} R T\right)=0 .
\end{array}
$$

Note that the radial vector component appears nowhere in these field equations, and will be determined algebraically using the field equation for the Lagrange multiplier field, $\lambda$. Substituting in the metric components, and solving the resulting equation for $A_{t}$ we have

$$
A_{t}=C_{2}+\frac{C_{3}}{r}
$$

where $C_{2}$ and $C_{3}$ are two more integration constants.

To determine the value of $C_{2}$ consider the $\lambda$ equation,

$$
\frac{A_{r}^{2}}{R}-\frac{A_{t}^{2}}{T}=-1,
$$

as $r \rightarrow \infty, A_{r}$ must be driven to zero so that isotropy is restored at spatial infinity. This expression therefore forces $A_{t}^{2} \rightarrow 1$, and so we find $C_{2}= \pm 1$. We choose the vector $A$ to be future pointing at spatial infinity, and so we pick $C_{2}=-1$. The value of $C_{3}$ can then be determined straightforwardly by substituting the expressions back into the Einstein equations. In particular, for the $(\theta, \theta)$ component we find

$$
\frac{Q^{2}}{r^{2}}=\frac{K C_{3}^{2}}{2 r^{2}}
$$

justifying our choice of the positive quantity $Q^{2}$. We then identify

$$
C_{3}=\sqrt{\frac{2}{K}} Q
$$

where $Q$ can be positive or negative. All that is left is to determine $A_{r}$ through the constraint equation (B14)

$$
\begin{aligned}
A_{r}= & \pm\left(1-\frac{2 M}{r}+\frac{Q^{2}}{r^{2}}\right)^{-1} \\
& \sqrt{\left(\frac{2}{K}-1\right) \frac{Q^{2}}{r^{2}}+\left(M+\sqrt{\frac{2}{K}} Q\right) \frac{2}{r}} .
\end{aligned}
$$

Phenomenologically $K<1$ [8], so the first term in the square root is positive. However at large $r$ the second term is dominant and is possibly negative. Thus for $A_{r}$ to be real we must satisfy the following bound

$$
M+\sqrt{\frac{2}{K}} Q \geq 0
$$

To summarise, 


$$
\begin{aligned}
g & =\operatorname{Diag}\left[-\left(1-\frac{2 M}{r}+\frac{Q^{2}}{r^{2}}\right),\left(1-\frac{2 M}{r}+\frac{Q^{2}}{r^{2}}\right)^{-1}, r^{2}, r^{2} \sin ^{2}(\theta)\right] \\
A_{t} & =-1+\sqrt{\frac{2}{K} \frac{Q}{r}} \\
A_{r} & = \pm\left(1-\frac{2 M}{r}+\frac{Q^{2}}{r^{2}}\right)^{-1} \sqrt{\left(\frac{2}{K}-1\right) \frac{Q^{2}}{r^{2}}+\left(M+\sqrt{\frac{2}{K}} Q\right) \frac{2}{r}}
\end{aligned}
$$

Thus the solution is Reissner-Nordström (RN), but where the gauge freedom for the field $A$ has been fixed up to a sign by the Lagrange multiplier constraint.

We have obtained a black hole solution in the EF metric. Its horizons are the ones observed by the gravitational components $g, A$, and $\phi$. Any matter fields are influenced by the MF metric (2) and it is important to consider the MF solution which is the observable frame.

\section{APPENDIX C: DETAILS OF NON-EXISTENCE ARGUMENT FOR BLACK HOLES WITH NON-CONSTANT SCALAR}

In this appendix we will argue that there are no black hole solutions 'near' to those found in section III A where the scalar is not constant. To support this hypothesis we first consider small perturbations to the scalar field about this constant scalar black hole solution. We only need consider one equation (C7). Since the background value for the scalar field is a constant, the equation of evolution for the scalar perturbation is simply

$$
\delta \phi^{\prime}=\frac{2 C_{1} K}{2 K(r-2) r+(K-1)\left(\frac{Q}{M}\right)^{2}-2 K \sqrt{\frac{2}{K}} \frac{Q}{M} r} .
$$

Unless $C_{1}=0$ this diverges for two values of $r$, which we denote $r_{\text {sing1 }}$ and $r_{\text {sing2 }}$ where $r_{\text {sing2 }} \geq r_{\text {sing1 }}$. The horizon positions are at $r_{+}$and $r_{-}$with $r_{+} \geq r_{-}$. It is straightforward to show that if $K>0$ and $-1 \leq \frac{Q}{M} \leq 1$ then $r_{\text {sing2 }}>r_{+}$and so this singularity will occur outside the outermost horizon. A scalar field singularity is usually a symptom of a singular geometry.

Of course, the linear theory breaks down as the scalar perturbations become large, and so while it indicates a singularity might form outside the horizon if we try to deform the scalar from being constant, it cannot be trusted. Hence we also solved numerically the full non-linear theory with asymptotic data that is close to that of the constant scalar RN solution, for particular parameters. The full numerical solutions confirm that indeed a naked singularity is found, as hinted at by the linear theory, with curvature invariants clearly diverging at the singular point. We now describe this in detail.
Using the same metric ansatz (B3) and the general form for the vector field (B2), the $r$-component of the vector field equation is

$$
A_{r}\left(\lambda+\frac{8 \pi\left(\phi^{\prime}\right)^{2}}{\kappa R}\right)=0
$$

and so for the case $A_{r} \neq 0$, this equation determines $\lambda$ to be proportional to the square of the proper derivative of $\phi$. Substituting $A_{r}$ from the constraint equation (B14), and this value of $\lambda$ into the other field equations yields Metric $(t, t)$-component

$$
\begin{array}{r}
-\frac{2 T}{r}+\frac{2 R T}{r}+\frac{2 T R^{\prime}}{R}-\frac{16 \pi r T\left(\phi^{\prime}\right)^{2}}{\kappa}= \\
K r\left(A_{t}^{\prime}\right)^{2}+\frac{8 \pi r A_{t}^{2}\left(\phi^{\prime}\right)^{2}}{\kappa} .
\end{array}
$$

Metric $(r, r)$-component

$$
\frac{2}{r}-\frac{2 R}{r}+\frac{2 T^{\prime}}{T}+\frac{K r\left(A_{t}^{\prime}\right)^{2}}{T}-\frac{16 \pi\left(\phi^{\prime}\right)^{2}}{\kappa}+\frac{8 \pi r A_{t}^{2}\left(\phi^{\prime}\right)^{2}}{\kappa T}=0 .
$$

Metric $(\theta, \theta)$-component

$$
\begin{gathered}
\frac{2 r T R^{\prime}}{R}-2 r T^{\prime}+\frac{r^{2} R^{\prime} T^{\prime}}{R}+\frac{r^{2}\left(T^{\prime}\right)^{2}}{T}+2 K r^{2}\left(A_{t}^{\prime}\right)^{2} \\
-\frac{32 \pi r^{2} T\left(\phi^{\prime}\right)^{2}}{\kappa}+\frac{16 \pi r^{2} A_{t}^{2}\left(\phi^{\prime}\right)^{2}}{r}-2 r^{2} T^{\prime \prime}=0 .
\end{gathered}
$$

Vector $t$-component

$$
K\left(-\frac{4}{r}+\frac{R^{\prime}}{R}+\frac{T^{\prime}}{T}\right) A_{t}^{\prime}+\frac{16 \pi A_{t}\left(\phi^{\prime}\right)^{2}}{\kappa}=2 K A_{t}^{\prime \prime}
$$

Scalar

$$
\phi^{\prime}=\frac{C_{1} \sqrt{R T}}{r^{2}\left(2 T-A_{t}^{2}\right)},
$$

where $C_{1}$ is an integration constant from the second order scalar field equation. This is normally associated with a scalar mass. Eliminating $A_{t}$ and $A_{t}^{\prime}$ between (C3) and (C4)

$$
\frac{16 \pi r\left(\phi^{\prime}\right)^{2}}{\kappa}=\frac{(T R)^{\prime}}{T R}
$$


and eliminating the same variables between (C5) and (C3), then substituting for $\phi^{\prime}$ using (C8) gives

$2 T\left(\frac{2(R-1)}{r}+\frac{R^{\prime}}{R}\right)+\left(\frac{r R^{\prime}}{R}-6\right) T^{\prime}+\frac{r\left(T^{\prime}\right)^{2}}{T}=2 r T^{\prime \prime}$,

which can be integrated once to obtain a first order equation in $T$

$$
R T\left(C_{2}+4 r^{2} T\right)=4 r^{2} T^{2}+4 r^{3} T T^{\prime}+r^{4}\left(T^{\prime}\right)^{2},
$$

where $C_{2}$ is a constant of integration. This equation allows elimination of one of the metric components $R$ from the system of equations to numerically integrate. Choosing to eliminate $R$ and $\phi^{\prime}$ using (C7), from (C8) and (C4), gives the system of equations

$$
\begin{aligned}
T^{\prime \prime} & =-\frac{2}{r\left(C_{2}+4 r^{2} T\right)}\left(\frac{C_{2} T}{r}+2\left(C_{2}+2 r^{2} T\right) T^{\prime}-r^{3} T^{\prime 2}-\frac{4 C_{1}^{2} \pi\left(2 T+r T^{\prime}\right)^{3}}{r \kappa\left(-2 T+A_{t}^{2}\right)^{2}}\right), \\
A_{t}^{\prime} & = \pm \sqrt{2}\left(\frac{\left(16 \pi C_{1}^{2}-\kappa C_{2}\left(2 T-A_{t}^{2}\right)\right)\left(T+r T^{\prime}\right)+r^{2}\left(4 \pi C_{1}^{2}+r^{2} \kappa\left(2 T-A_{t}^{2}\right)\right)\left(T^{\prime}\right)^{2}}{r^{2} \kappa K\left(C_{2}+4 r^{2} T\right)\left(2 T-A_{t}^{2}\right)}\right)^{\frac{1}{2}} .
\end{aligned}
$$

From which $\phi^{\prime}$ can be obtained during the numerical integration through the scalar field equation (C7).

We then integrate inwards from large $r$, imposing an asymptotically flat spacetime, a constant scalar and vanishing $A_{r}$ component at spatial infinity. Looking first to the asymptotic expansion of $T$ and $A_{t}$ will allow identification of the free parameters available for the boundary condition at large $r$. Assuming the general asymptotic form

$$
\begin{aligned}
T(r) & =t_{0}+\frac{t_{1}}{r}+\frac{t_{2}}{r^{2}}+\mathcal{O}\left(\frac{1}{r^{3}}\right), \\
A_{t}(r) & =u_{0}+\frac{u_{1}}{r}+\frac{u_{2}}{r^{2}}+\mathcal{O}\left(\frac{1}{r^{3}}\right) .
\end{aligned}
$$

We may use the freedom in rescaling the $t$ coordinate in setting $t_{0}=1$, and the freedom in the $r$ coordinate to set $t_{1}$ to -1 . Note that this explicitly sets the standard RN mass to be positive. It then follows from the constraint equation that if we are to have $A_{r} \rightarrow 0$ as $r \rightarrow \infty$ then $A_{t} \rightarrow-1$ and $u_{0}=-1$. The remaining coefficients may be determined by performing a series expansion about infinity of the two differential equations (C11C12). We find

$$
\begin{aligned}
T & \simeq 1-\frac{1}{r}+\frac{1+\frac{32 \pi C_{1}^{2}}{\kappa}-C_{2}}{4 r^{2}} \\
A_{t} & \simeq-1+ \pm \sqrt{\frac{2}{K}} \frac{\sqrt{1+\frac{16 \pi C_{1}^{2}}{\kappa}-C_{2}}}{2 r}-\frac{4 \pi C_{1}^{2}}{\kappa K} \frac{1}{r^{2}}
\end{aligned}
$$

where the sign choice comes from the sign of the gradient of $A_{t}$, equation (C12). There is only one consistent choice for this, as may be seen by using the Lagrange multiplier equation (B14) to calculate the corresponding asymptotic expansion for $A_{r}^{2}$. To lowest order:

$$
A_{r}^{2}=\left[1- \pm \sqrt{\frac{2}{K}} \sqrt{1+\frac{16 \pi C_{1}^{2}}{\kappa}-C_{2}}\right] \frac{1}{r}+\mathcal{O}\left(\frac{1}{r^{2}}\right) .
$$

Thus for the reality of $A_{r}$ we are forced to choose the negative sign.

We are now left with four parameters: two constants, $\kappa$ and $K$ and two free parameters $C_{1}$ and $C_{2}$ with an additional condition to ensure the reality of $A_{t}$

$$
1+\frac{16 \pi}{\kappa} C_{1}^{2}-C_{2}>0
$$

Additionally, one can identify these parameters with the standard Reissner-Nordström charge to mass ratio,

$$
\left(\frac{Q}{M}\right)^{2} \equiv 1-C_{2}
$$

and a quantity associated with the scalar

$$
S^{2} \equiv \frac{16 \pi C_{1}^{2}}{\kappa}
$$

so that the reality condition (C18) is now guaranteed. Using this definition of $S$, the differential equations to numerically integrate $\mathrm{C} 11 \mathrm{C} 12$ are reduced to a constant $K$, and the two parameters $S$ and $Q / M$.

Naively considering the $\phi$ equation of motion (C7), one sees that a possible divergence occurs when $2 T=A_{t}^{2}$. This does occur, and we demonstrate below for the parameter choices $K=0.01, \kappa=0.01, Q / M=0.01$ and a small scalar charge, $S=0.001$.

We find that both the EF and MF Ricci scalars diverge, and thus it is not a coordinate singularity - this is shown in the left panel of figures 14 and 15. Further, at no point outside of the singular position does the $g_{t t}$ component of 


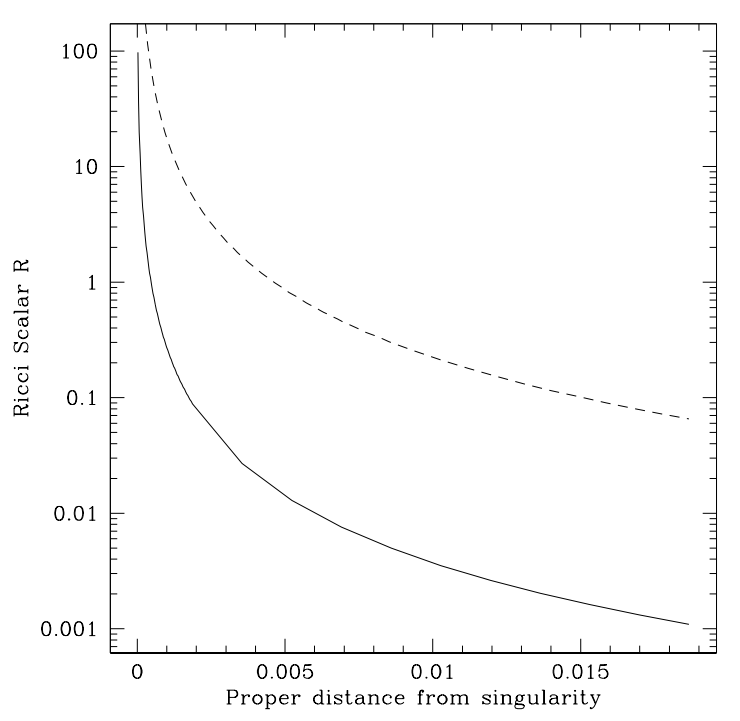

FIG. 14: EF (solid) and MF (dashed) Ricci Scalars for the parameters $S=0.001$ and $K=\kappa=Q / M=0.01$ as functions of the proper distance from the singular surface, illustrating a physical singularity.

the metric vanish - the singularity is not enclosed within a horizon. $\phi, T$ and $A_{t}$ remain finite (though their proper gradients diverge) up to the singular point. Interestingly, at the singular point $g_{r r} \equiv R=0$ implying (through $2 T-A_{t}^{2}=0$ and $A^{2}=-1$ ) that the radial component of the TeVeS vector vanishes at this point.

We have shown that linear theory suggests no deformation of the constant scalar RN solution that has regular horizon, and non-trivial scalar. In specific cases we have confirmed that the full non-linear theory agrees with the linear theory in that attempts to make the scalar be nonconstant lead to a naked singularity rather than a regular horizon. However, we have not shown that there is no such solution far in 'solution-space' from the constant scalar RN solution. We think it unlikely, although have not explored this possibility in detail.

\section{APPENDIX D: DETAILS OF FULL DYNAMICAL NUMERICAL SIMULATION METHOD}

We use the following Schwarzschild-like coordinate system for our evolution,

$$
d s^{2}=-T^{2}(t, r) d t^{2}+e^{R(t, r)} d r^{2}+r^{2} d \Omega_{2}^{2} .
$$

In this coordinate system the Einstein equations have the usual constraints (the $t t$ and $t r$ components) together with a second order evolution equation for $R$, the $\theta \theta$ component. We do not use this directly to evolve $R$, instead we use the $\operatorname{tr}$ constraint equation itself. Once all variables (apart from $T$ ) have been successfully evolved to

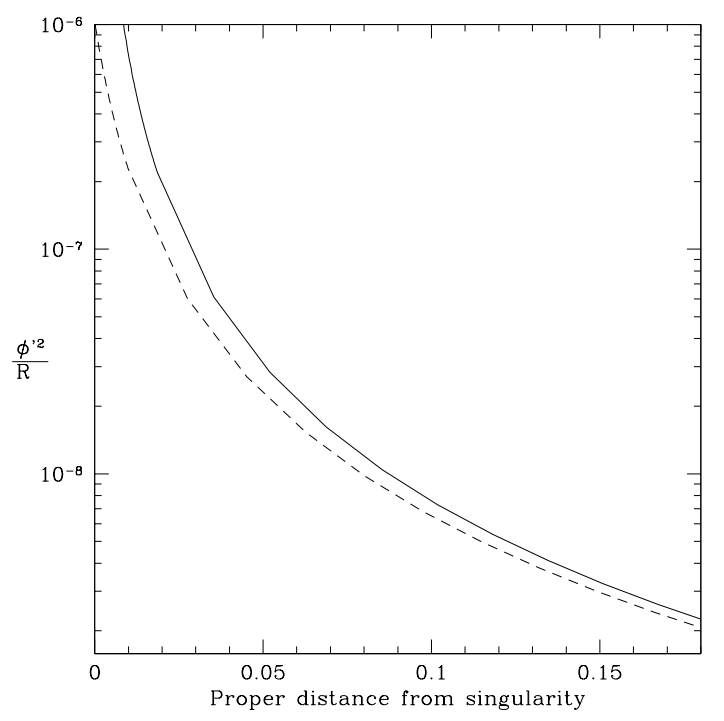

FIG. 15: Square of the proper derivative of $\phi, \frac{\phi^{\prime 2}}{R}$, for the full integration (solid) and for the linearised theory (dashed). A larger $S$ would see a larger discrepancy between the singular positions for the full and the linearised theory. This plot also demonstrates the agreement of the two curves away from the singularity.

the next spatial slice, we integrate across the grid in the radial direction to obtain $T$ on the slice using the $r r$ equation. A simplification is made for this radial integration of $T$; we use the value of $T$ from the old spatial slice to compute the contribution to the right hand side of the $r r$-Einstein equation, for the sake of computational run time. Using this approximation, the $r r$ component is a first order differential equation in $T$.

We find that the most stable way to evolve the vector field is to evolve $\lambda$, rather than evolving $A$ and then calculating $\lambda$ through contraction of the vector field equation. The evolution equation we use for $\lambda$ is given by taking the divergence of the vector equation

$$
\nabla_{\alpha} \nabla_{\beta} F^{\beta \alpha}+\nabla_{\alpha}\left(\lambda A^{\alpha}\right)+\nabla_{\alpha}\left(\sigma^{2} A^{\beta} \phi, \beta g^{\alpha \gamma} \phi, \gamma\right)=\nabla_{\alpha}\left[\left(1-e^{-4 \phi}\right) g^{\alpha \mu} A^{\beta} \tilde{T}_{\mu \beta}\right]
$$


The first term vanishes through antisymmetry of $F$ and the symmetry of the Ricci Tensor. The third term will contain second time derivatives of $\phi$, and so we substitute for this using the $\phi$ equation of motion. The last term will contain second derivatives of the $\chi$ field. For this we do not substitute from the $\chi$ field equation as we are able to use the conservation properties of $\tilde{T}$ to obtain a simpler (and so numerically advantageous) expression. Consider the definition of $\tilde{T}$ from a variation of the action with respect to the MF metric,

$$
\delta S=-\frac{1}{2} \tilde{T}_{\alpha \beta} \sqrt{\tilde{g}} \delta \tilde{g}^{\alpha \beta},
$$

and one may rewrite the variation of $\tilde{g}$ in terms of variations of the other fields, through the disformal relation 2] (see [8])

$$
\delta \tilde{g}^{\alpha \beta}=e^{2 \phi} \delta g^{\alpha \beta}+2 \sinh 2 \phi A_{\mu} \delta g^{\mu(\alpha} A^{\beta)}+2\left[e^{2 \phi} g^{\alpha \beta}+2 A^{\alpha} A^{\beta} \cosh 2 \phi\right] \delta \phi+2 \sinh 2 \phi A^{(\alpha} g^{\beta) \mu} \delta A_{\mu}
$$

Specifically, consider the case of a diffeomorphism generated by the vector field itself,

$$
\begin{aligned}
\delta g^{\alpha \beta}=£_{A} g^{\alpha \beta} & =\nabla^{(\alpha} A^{\beta)} \\
\delta A^{\mu}=£_{A} A^{\mu} & =0 \\
\delta \phi=£_{A} \phi & =A^{\alpha} \nabla_{\alpha} \phi
\end{aligned}
$$

so that

$$
\delta A_{\nu}=\delta\left(g_{\nu \mu} A^{\mu}\right)=-A_{\alpha} g_{\nu \beta} \delta g^{\alpha \beta}=-A_{\alpha} g_{\nu \beta} \nabla^{(\alpha} A^{\beta)} .
$$

Inserting all of this into (D3) and integrating by parts from the terms containing variations of the inverse EF metric, we obtain the following expression

$$
A^{\beta} \nabla^{\alpha} \tilde{T}_{\alpha \beta}=\left(g^{\alpha \beta}+\left(1+e^{-4 \phi}\right) A^{\alpha} A^{\beta}\right) A^{\mu} \nabla_{\mu} \phi,
$$

which we can then use instead of the $\chi$ evolution equation in the evolution of $\lambda$.

Thus both the metric components and $\lambda$ are evolved with first order differential equations, whilst $A, \phi$ and $\chi$ are evolved at second order. The origin boundary conditions are $\phi,{ }_{r}=0, A^{r}=0, T=1, R=0, T,_{r}=0, R,_{r}=0$ and $\chi,{ }_{r}=0$.

For the scalar shell collapse in section (IVA) the initial conditions on the $t=0$ spatial slice, are $\phi=\phi_{c}, A^{r}=0$, $R,,_{t}=T,_{t}=\phi,{ }_{t}=\chi,_{t}=0$. We consider two simulations with differing initial conditions for $F_{\mu \nu}$, and these are discussed in the text. The initial $R$ and $T$ configurations are specified by solving the $r r$ and $t t$ Einstein equations for a choice of Gaussian initial data on $\chi$. The $t r$-Einstein equation is automatically satisfied for this initial data.

For the boson star in section (IVB) the initial conditions for all fields are fixed by requiring for a static star configuration initially. This configuration was found using a radial shooting method, as discussed in the text.

Second order finite differencing was used to discretize the equations of motion. For the scalar-shell collapse simulation, we used resolutions up to $\Delta r=0.05$ and $\Delta t=0.0005$, whilst for the Boson star simulation we used resolutions up to $\Delta r=0.05$ and $\Delta t=0.00005$. Each simulation had 400 simulation sites per slice, and took on the order of a day to run using an average desktop computer.

\section{APPENDIX E: CONSTRAINT TESTING}

We ran simulations at a variety of resolutions to check convergence which was seen in accord with our second order finite differencing. To test the constraint equations (and convergence), we consider the black hole dynamics for some representative initial data as in section IVA, and take the absolute value of the constraint equation (l.h.s. - r.h.s.) at a grid point, and then average this over all grid points (labelled by $i$ ) in a large physical area. We then compute this average for various spatial and temporal resolutions, keeping the physical region fixed. We denote the set of all grid points in this region as $\sigma$. Figure [16] shows this sum for the $t t$ component of the Einstein equation against $\log _{10} \Delta t$, for four spatial steps $\Delta r=0.2$, $0.1,0.05$ and 0.025 . We clearly see agreement with expected constraint behaviour for second order differencing as the spatial and temporal resolutions are reduced. Similar checks were performed for the boson star simulation of section IVB.
[1] C. B. Netterfield, P. A. R. Ade, J. J. Bock, J. R. Bond, J. Borrill, A. Boscaleri, K. Coble, C. R. Contaldi, B. P. Crill, P. de Bernardis, et al., ApJ 571, 604 (2002), arXiv:astro-ph/0104460.
[2] N. W. Halverson, E. M. Leitch, C. Pryke, J. Kovac, J. E. Carlstrom, W. L. Holzapfel, M. Dragovan, J. K. Cartwright, B. S. Mason, S. Padin, et al., ApJ 568, 38 (2002), arXiv:astro-ph/0104489. 


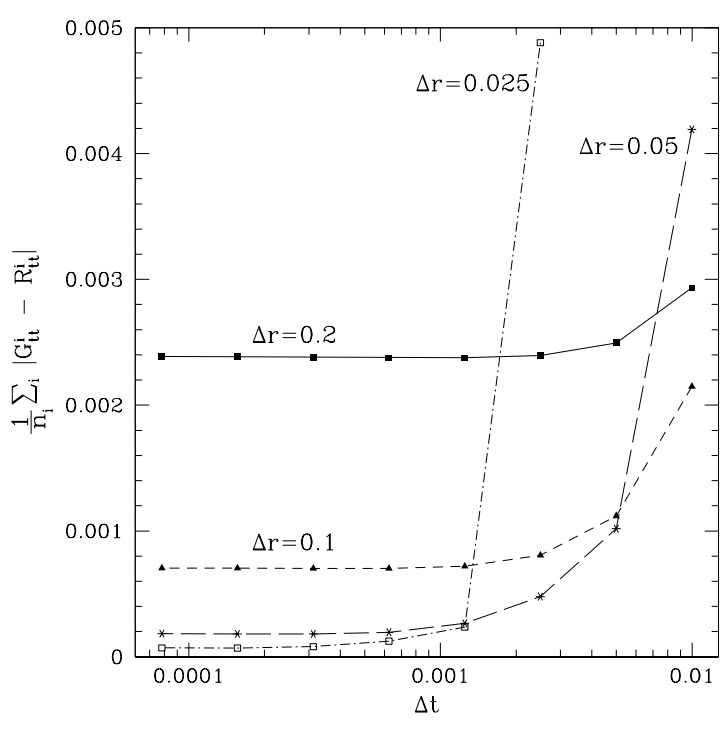

FIG. 16: The average of the $t t$ constraint equation over simulated grid points for the black hole of section IV A for different time steps. Each curve corresponds to a different spatial resolution.

[3] T. J. Pearson, B. S. Mason, A. C. S. Readhead, M. C. Shepherd, J. L. Sievers, P. S. Udomprasert, J. K. Cartwright, A. J. Farmer, S. Padin, S. T. Myers, et al., ApJ 591, 556 (2003), arXiv:astro-ph/0205388.

[4] G. Hinshaw, D. N. Spergel, L. Verde, R. S. Hill, S. S. Meyer, C. Barnes, C. L. Bennett, M. Halpern, N. Jarosik, A. Kogut, et al., ApJS 148, 135 (2003), arXiv:astro$\mathrm{ph} / 0302217$.

[5] D. Clowe, M. Bradač, A. H. Gonzalez, M. Markevitch, S. W. Randall, C. Jones, and D. Zaritsky, ApJ 648, L109 (2006), arXiv:astro-ph/0608407.

[6] M. Milgrom, ApJ 270, 365 (1983).

[7] R. H. Sanders and S. S. McGaugh, ARAA 40, 263 (2002), arXiv:astro-ph/0204521.

[8] J. D. Bekenstein, Phys. Rev. D70, 083509 (2004), astro$\mathrm{ph} / 0403694$.

[9] J. D. Bekenstein and R. H. Sanders, ApJ 429, 480 (1994), arXiv:astro-ph/9311062.

[10] R. H. Sanders, ApJ 480, 492 (1997), arXiv:astro$\mathrm{ph} / 9612099$.

[11] H. Zhao, ArXiv Astrophysics e-prints (2006), astro$\mathrm{ph} / 0611777$.

[12] C. Skordis, Phys. Rev. D74, 103513 (2006), astro$\mathrm{ph} / 0511591$.

[13] S. Dodelson and M. Liguori, Physical Review Letters 97, 231301 (2006), arXiv:astro-ph/0608602.

[14] F. Bourliot, P. G. Ferreira, D. F. Mota, and C. Skordis, Phys. Rev. D 75, 063508 (2007), arXiv:astro$\mathrm{ph} / 0611255$.

[15] C. Skordis (2008), arXiv:0801.1985 [astro-ph].

[16] G. W. Angus, H. Y. Shan, H. S. Zhao, and B. Famaey, ApJ 654, L13 (2007), arXiv:astro-ph/0609125.

[17] D. Giannios, Phys. Rev. D71, 103511 (2005), grqc/0502122.

[18] J. Bekenstein and J. Magueijo, Phys. Rev. D73, 103513
(2006), astro-ph/0602266.

[19] T. Jacobson and D. Mattingly, Phys. Rev. D64, 024028 (2001), gr-qc/0007031.

[20] M. A. Clayton (2001), gr-qc/0104103.

[21] N. Arkani-Hamed, H.-C. Cheng, M. A. Luty, and S. Mukohyama, JHEP 05, 074 (2004), hep-th/0312099.

[22] N. Arkani-Hamed, H.-C. Cheng, M. A. Luty, S. Mukohyama, and T. Wiseman, JHEP 01, 036 (2007), hep$\mathrm{ph} / 0507120$.

[23] V. A. Kostelecky and S. Samuel, Phys. Rev. D40, 1886 (1989).

[24] E. Sagi and J. D. Bekenstein, Phys. Rev. D77, 024010 (2008), arXiv:0708.2639 [gr-qc].

[25] N. E. Mavromatos (2007), arXiv:0708.2250 [hep-th].

[26] N. Mavromatos and M. Sakellariadou, Phys. Lett. B652, 97 (2007), hep-th/0703156.

[27] C. Bonvin, R. Durrer, P. G. Ferreira, G. Starkman, and T. G. Zlosnik (2007), arXiv:0707.3519 [astro-ph].

[28] T. G. Zlosnik, P. G. Ferreira, and G. D. Starkman, Phys. Rev. D75, 044017 (2007), astro-ph/0607411.

[29] R. H. Sanders, Mon. Not. Roy. Astron. Soc. 363, 459 (2005), astro-ph/0502222.

[30] J.-P. Bruneton, Phys. Rev. D75, 085013 (2007), grqc/0607055.

[31] T. Jacobson (2008), arXiv:0801.1547 [gr-qc].

[32] M. D. Seifert, Phys. Rev. D76, 064002 (2007), grqc/0703060.

[33] M. D. Seifert and R. M. Wald, Phys. Rev. D75, 084029 (2007), gr-qc/0612121.

[34] R. Ruffini and S. Bonazzola, Phys. Rev. 187, 1767 (1969).

[35] M. Gleiser, Phys. Rev. D 38, 2376 (1988).

[36] B. Z. Foster, Phys. Rev. D72, 044017 (2005), grqc/0502066.

[37] G. D. Moore and A. E. Nelson, JHEP 09, 023 (2001), hep-ph/0106220.

[38] J. W. Elliott, G. D. Moore, and H. Stoica, JHEP 08, 066 (2005), hep-ph/0505211.

[39] T. Koivisto and D. F. Mota (2008), arXiv:0801.3676 [astro-ph].

[40] D.-M. Chen (2007), arXiv:0712.1633 [astro-ph].

[41] D. Xu et al. (2007), arXiv:0710.4935 [astro-ph].

[42] M. Milgrom and R. H. Sanders (2007), arXiv:0709.2561 [astro-ph].

[43] M. Feix, C. Fedeli, and M. Bartelmann (2007), arXiv:0707.0790 [astro-ph].

[44] F. Schmidt, M. Liguori, and S. Dodelson, Phys. Rev. D76, 083518 (2007), arXiv:0706.1775 [astro-ph].

[45] P. Zhang, aff, R. Bean, and S. Dodelson (2007), arXiv:0704.1932 [astro-ph].

[46] R. Takahashi and T. Chiba, Astrophys. J. 671, 45 (2007), astro-ph/0701365.

[47] B. Famaey, G. Gentile, J.-P. Bruneton, and H.-S. Zhao, Phys. Rev. D75, 063002 (2007), astro-ph/0611132.

[48] O. Bertolami and J. Paramos (2006), gr-qc/0611025.

[49] F. Bourliot, P. G. Ferreira, D. F. Mota, and C. Skordis, Phys. Rev. D75, 063508 (2007), astro-ph/0611255.

[50] D.-M. Chen and H. Zhao (2006), astro-ph/0606506.

[51] D.-M. Chen and H.-S. Zhao, Astrophys. J. 650, L9 (2006).

[52] X.-h. Jin and X.-z. Li (2006), gr-qc/0605046.

[53] L. M. Diaz-Rivera, L. Samushia, and B. Ratra, Phys. Rev. D73, 083503 (2006), astro-ph/0601153.

[54] H. Zhao (2005), astro-ph/0508635. 
[55] M.-C. Chiu, C.-M. Ko, and Y. Tian, Astrophys. J. 636, 565 (2006), astro-ph/0507332.

[56] C. Skordis, D. F. Mota, P. G. Ferreira, and C. Boehm, Phys. Rev. Lett. 96, 011301 (2006), astro-ph/0505519.

[57] H.-S. Zhao, D. J. Bacon, A. N. Taylor, and K. Horne, Mon. Not. Roy. Astron. Soc. 368, 171 (2006), astro$\mathrm{ph} / 0509590$.

[58] A. Slosar, A. Melchiorri, and J.I. Silk, Phys. Rev. D72, 101301(R) (2005), astro-ph/0508048.

[59] C. Eling and T. Jacobson, Class. Quant. Grav. 23, 5643 (2006), gr-qc/0604088.

[60] C. Eling and T. Jacobson, Class. Quant. Grav. 23, 5625 (2006), gr-qc/0603058.

[61] C. Eling, B. Z. Foster, T. Jacobson, and A. C. Wall, Phys. Rev. D75, 101502(R) (2007), hep-th/0702124.

[62] It is interesting that the analytic analysis here does actually hold for exactly constant scalar - the function $\mathcal{F}$ vanishes in this limit. However that is not the physical regime we are interested in. In any physical context there will always be some small scalar gradients.

[63] Note that in [24] the authors derive a RN solution in the MF by introducing a 'true' Maxwell field. Their charge is therefore unrelated to the charge $Q$ in our solution.

[64] Giannios [17] and more recently, Sagi \& Bekenstein [24] have found black hole solutions where the scalar diverges logarithmically, but the MF metric remains regular. Note that these solutions are not 'near' ours in the sense that the vector field has a very different behaviour as we have discussed in this section.

[65] We thank Bekenstein for emphasizing this in private communication.

[66] In pure TeVeS there are no connection terms appearing in the expression for $\Theta_{\mu \nu}$ above and so this does not introduce second derivatives of $A$ on the right hand side of Einstein's equations, and is advantageous for solving the initial-value problem. This is one reason Bekenstein gives for originally making this special choice of vector action. Unfortunately this is a luxury not afforded by the modified theory, which is not a problem in principle, but does complicate a treatment of the initial value problem. Hence we have not yet attempted to reproduce the numerical dynamical calculations of the previous section in the modified theory, although it would be interesting to do so. 